\title{
Langevin simulations of the out-of-equilibrium dynamics of the vortex glass in high-temperature superconductors
}

\author{
Sebastian Bustingorry, ${ }^{1}$ Leticia F. Cugliandolo, ${ }^{2}$ and Daniel Domínguez ${ }^{1}$ \\ ${ }^{1}$ Centro Atómico Bariloche, 8400 San Carlos de Bariloche, Río Negro, Argentina \\ 2 Université Pierre et Marie Curie - Paris VI, LPTHE UMR 7589, 4 Place Jussieu, 75252 Paris Cedex 05, France \\ and LPT Ecole Normale Supérieure, 24 rue Lhomond, 75231 Paris Cedex 05 France
}

(Dated: September 2, 2018)

\begin{abstract}
We study the relaxation dynamics of flux lines in dirty high-temperature superconductors using numerical simulations of a London-Langevin model of the interacting vortex lines. By analysing the equilibrium dynamics in the vortex liquid phase we find a dynamic crossover to a glassy non-equilibrium regime. We then focus on the out-of-equilibrium dynamics of the vortex glass phase using tools that are common in the study of other glassy systems. By monitoring the two-times roughness and dynamic wandering we identify and characterize finite-size effects that are similar, though more complex, than the ones found in the stationary roughness of clean interface dynamics. The two-times density-density correlation and mean-squared-displacement correlation age and their temporal scaling follows a multiplicative law similar to the one found at criticality. The linear responses also age and the comparison with their associated correlations shows that the equilibrium fluctuationdissipation relation is modified in a simple manner that allows for the identification of an effective temperature characterizing the dynamics of the slow modes. The effective temperature is closely related to the vortex liquidvortex glass crossover temperature. Interestingly enough, our study demonstrates that the glassy dynamics in the vortex glass is basically identical to the one of a single elastic line in a disordered environment (with the same type of scaling though with different parameters). Possible extensions and the experimental relevance of these results are also discussed.
\end{abstract}

PACS numbers: 74.25.Qt, 64.70.Pf, 61.20.Lc, 74.25.Sv

\section{INTRODUCTION}

Vortex matter in high-temperature superconductors has been extensively studied for many years $1.2,3.4$ In particular, the nature of the dynamics of interacting vortices in their different phases has attracted much attention due to its relevance in technological applications.

Most studies of vortex systems have focused on the analysis of their static properties. It is well known that at low temperatures any small amount of disorder destroys the long range order of the Abrikosov lattice, ${ }^{5}$ leading to different glassy phases. For small magnetic field, i.e. small vortex density, quasi-long range order is retained in the so-called Bragg glass (BG) 6,7,8,9 The key characteristics of the BG are the logarithmic decay of spatial correlations and the absence of dislocations, giving rise to a well defined structure factor. For increasing fields, the appearance of dislocation loops signals the transformation to a new disordered phase, characterized by the rapid destruction of Bragg peaks observed in neutron scattering ${ }^{7}$ This is the so-called vortex glass $(\mathrm{VG}){ }^{10.11}$ Increasing the temperature both glassy phases transform into a vortex liquid (VL). Transport and magnetic measurements showed that at low-fields the BG solid melts into the VL through a first order transition, while the transition from the VG solid to the VL at high fields is continuous 12.13 Whether the latter corresponds to a true phase transition or a crossover is still under debate. $14,15,16,17,18,19,20,21,22,23,24$

Beyond the nature of the continuous VG-VL transition, important efforts were devoted to the interpretation of the irreversibility line (IRL) ${ }^{25.26 .27}$ Experimentally, the IRL marks both the onset of the irreversibility in zero field cooling and field cooling magnetization measurements and the separation of two transport regimes in the field-temperature diagram. Above the IRL, thermal energy dominates, vortices are unable to be pinned, and any amount of current results in a linear current-voltage characteristics. Below the IRL, the pinning energy dominates and flux lines are irreversibly pinned. In this case, the resistivity drops to nearly zero with a nonlinear current-voltage characteristics. Originally, the proposition that the IRL signals the continuous VG-VL phase transition was supported by experiments showing the scaling of the current-voltage characteristic, ${ }^{28.29}$ and by simulations in randomly frustrated 3D XY models without screening showing evidence for a finite temperature critical point. ${ }^{14,20,30}$ However, contradictions in the experimental scaling of the currentvoltage characteristics, ${ }^{29.31 .32}$ on the one hand, and the disappearance of the finite temperature transition in the randomly frustrated 3D XY model when screening is restored, 15.16 .17 .18 on the other hand, have raised new questions on how to interpret the IRL.

Based on the re-examination of experimental data and the results of numerical simulations of the over-damped dynamics of the London-Langevin model, Reichhart et al ${ }^{21}$ argued that the vortex-glass criticality is arrested at a crossover temperature. Below this temperature, the relaxation times as obtained from the study of the resistivity grow very quickly in a way that is consistent with the Vogel-Fulcher law commonly found in relaxation studies of structural glasses. ${ }^{33}$ This study suggests that the VL and the VG are then just separated by a crossover phenomenon in which relaxation times go beyond the experimental time-window at a glass temperature $T_{g}(H)$. Below this crossover line one expects to find an outof-equilibrium system, the VG, with all the properties of more standard glasses. 
In the last decade there has been important progress in the understanding of the out-of-equilibrium dynamics of glasses. A key characteristic of relaxing glassy systems is the loss of stationarity reflected by their aging properties, meaning that the system's dynamics depend on the time elapsed after the preparation of the sample, ${ }^{34} t_{w}$. As a consequence, dynamic correlation functions dependend now on two times, the "waiting time", $t_{w}$, and the time $t$ elapsed during the measurement. Also the linear response functions of glassy systems show aging effects, being dependent on $t_{w}$ and $t$, and they are anomalous in the sense that they are not related to their associated correlation functions by the equilibrium fluctuation-dissipation theorem (FDT). The relation between the two functions, correlation and response, remains, however, rather simple. It has been characterized in a number of glassy systems, allowing for a kind of classification of the out-ofequilibrium dynamics of disordered systems. ${ }^{35.36}$ The comparison between linear response and correlation has shed light onto the role played by different degrees of freedom in the relaxation of the full system. It allowed for the identification of an effective temperature in these non-equilibrium systems with slow dynamics ${ }^{37}$ For example, the violation of the FDT was studied numerically in driven vortex lattices with random pinning. 38

Metastability and aging-like phenomena have been observed in the nonlinear transport in the $\mathrm{VG}$ state of single crystal $\mathrm{Bi}_{2} \mathrm{Sr}_{2} \mathrm{CaCu}_{2} \mathrm{O}_{8}$ samples ${ }^{39.40}$ and analyzed in numerical simulations ${ }^{41.42}$ Also, history dependent effects have been found in $\mathrm{YBa}_{2} \mathrm{Cu}_{3} \mathrm{O}_{7}$ near the peak effect. ${ }^{43}$ Aging phenomena has been reported in the magnetization of granular $\mathrm{Bi}_{2} \mathrm{Sr}_{2} \mathrm{CaCu}_{2} \mathrm{O}_{8}$ samples at low fields. ${ }^{44.45}$ However, in this case, the effect is due to a chiral glass phase that is found in three-dimensional Josephson junctions networks with $\pi$ junctions, ${ }^{46}$ thought to model granular high- $T_{c}$ superconductors. Theoretically, Nicodemi and Jensen studied a simplified two-dimensional lattice Hamiltonian model for the effective vortex dynamics to address the issue of equilibrium and out-of-equilibrium dynamics. ${ }^{47}$ They focused on the magnetization relaxation using both one-time and twotimes correlation functions. They observed that relaxation times dramatically increase upon decreasing temperature, with a Vogel-Fulcher-like dependence, and established some analogies with glass formers and supercooled liquid systems. This two-dimensional model emphasizes the effect of random quenched disorder but overlooks the importance of the threedimensionality of flux lines and their fluctuations along the axis parallel to the magnetic field. Other studies focused on the out-of-equilibrium features of vortex creep motion at low temperatures. 48,49

In the present work, we compare the dynamics of vortex matter in high-temperature superconductors with that of structural glasses aiming at clarifying the nature of the VG phase. We extend and complement our previous study of the out-ofequilibrium dynamics of a London-Langevin model ${ }^{50}$ Extensive numerical simulation of the over-damped dynamics were performed to study correlation functions such as the roughness of the flux lines, their dynamic wandering, the structure factor and mean-square displacement all generalized to in-

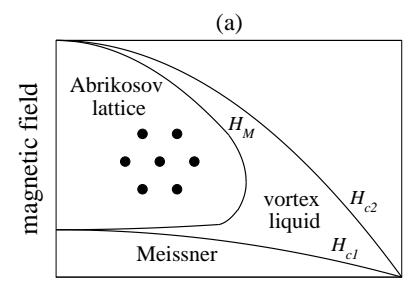

temperature

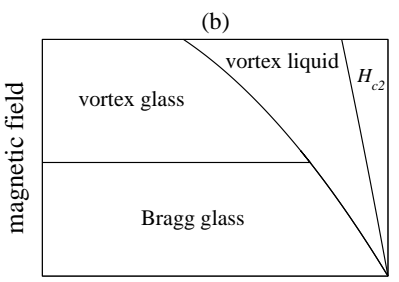

temperature
FIG. 1: Sketch of the vortex phase diagram in high-temperature superconductors for (a) clean and (b) dirty samples. In (a) the Abrikosov lattice phase, prototype of the vortex solid, is located between the two critical magnetic fields, $H_{c 1}$ and $H_{c 2}$, at low temperatures. At high temperatures the Abrikosov lattice melts into a vortex liquid phase at $H_{M}(T)$. Below $H_{c 1}$ the Meissner phase without vortex is found. In (b) the Meissner phase at very low magnetic field is not shown and the three phases, Bragg glass, vortex glass and vortex liquid, are sketched.

clude two-times dependencies. Focusing on the mean-square displacement and its conjugated response function we show that the scaling of both functions is multiplicative and we analyze how this is related to a non-trivial violation of the FDT ${ }^{50}$

The outline of the paper is as follows. In Sec. I a brief description of the vortex phase diagram and glassy properties is presented. Section 1 gives an elaborated description of the model and details on the numerical simulations. The quantities of interest for the present study are presented in Sec.IV In Sec. D we show how the VG phase is approached both from the structural and dynamical properties. Finite size effects are discussed in Sec. DI The aging behavior of flux lines in the vortex glass and their temperature dependence is presented in Sec.VII The multiplicative scaling of aging correlations and responses is described in Sec.VIII the violation of the FDT, the effective temperature and its relationship with the the dynamical arrest of the VL diffusion are discussed. Finally, Sec. IX is devoted to the discussion of our results.

\section{BACKGROUND}

In this Section we present the general background on which we base our study. Our aim is to give here a brief review of the vortex matter phases in high-temperature superconductors as well as a description of the main features of the nonequilibrium relaxation in glassy systems and the violation of FDT

\section{A. Vortex phases in high-temperature superconductors}

Several review articles describe the physics of vortex matter. $1,2,3,4$ Here we briefly summarize the main characteristics of vortex phases in high-temperature superconductors, referring the reader to these specialized reviews 1.2 .3 .4 for further details.

In type II superconductors, below a lower critical field $H_{c 1}$, the Meissner effect does not allow the magnetic field to 
penetrate the superconductor sample. Above $H_{c 1}$, the magnetic field penetrates into the superconductor sample, continuously increasing the flux penetration until the upper critical field $H_{c 2}$ where the sample becomes normal, see Fig. 11 The magnetic field penetrates the superconductor under the form of flux lines (or vortices) each one carrying a flux quantum $\Phi_{0}=h c / 2 e$. Vortices are composed by an inner normal core of radius $\xi$ (the coherent length), surrounded by super-current screening over a distance $\lambda$ (the penetration length). In the absence of disorder and at low temperatures the vortices form a triangular crystal, the so-called Abrikosov lattice,, 51.52 .53 due to the repulsive vortex-vortex interaction. At high temperature thermal fluctuations melt the Abrikosov lattice into a vortex liquid (VL) phase when the magnetic field $H_{M}<H_{c 2}$ is reached. In the VL vortices move with a non-zero diffusion constant, leading to a linear current-voltage characteristics and dissipation. These observations have led to the phase diagram in Fig 11a). The re-entrant behavior of the melting line $H_{M}(T)$ originates in the drastic softening of the elastic constants of the Abrikosov lattice when the vortex density is lowered close to $H_{c 1}$.

The high-temperature superconductors reveal new vortex physics phenomena related to the relevance of thermal fluctuations and disorder in these systems. A schematic representation of a vortex phase diagram, when disorder effects are included, in high-temperature superconductors is presented in Fig 1 b) 19.54 This type of phase diagram is experimentally observed in layered systems like $\mathrm{YBa}_{2} \mathrm{Cu}_{3} \mathrm{O}_{7-x}(\mathrm{YBCO}){ }^{55}$ and $\mathrm{Bi}_{2} \mathrm{Sr}_{2} \mathrm{CaCu}_{2} \mathrm{O}_{8+s} .56$ In Fig 1 b) the Meissner phase at very low magnetic field is not shown. At high temperature thermal fluctuations dominate and the VL is observed. At low temperature disorder dominates and, when it is short-ranged correlated, two glassy phases are observed: the Bragg glass $(\mathrm{BG})^{57}$ at low magnetic field and the vortex glass (VG) at high magnetic field. The BG presents a structure factor with well defined intensity Bragg peaks at the reciprocal wave vectors of the triangular lattice. Long-range order, however, is destroyed by disorder leading to quasi-long range order with spatial correlations decaying logarithmically and power-law diverging Bragg peaks. .9 At high temperature the BG melts into the VL phase through a first-order transition analogously to the melting of the Abrikosov lattice. Upon increasing magnetic field or disorder, at low temperatures, the BG transforms into the vortex glass (VG) also through a first order transition. $54,58,59$

In the VG phase scenario, disorder destroys long-range order leading to a short-range amorphous structure. ${ }^{10.11}$ While the high-temperature VL can be considered to be made of mobile vortices moving unhindered over the pinning potential, the low temperature VG is thought to be an immobile amorphous solid with the flux lines localized on the pinning centers in order to minimize the pinning energy. The VG theory predicts that collective effects are able to produce infinite energy barriers at low temperatures, leading to a strictly zero fluxflow resistance with no dissipation. ${ }^{10.11}$ This leads to interesting scaling theories for the energies involved in creep motion of flux lines. ${ }^{49,60,61}$ Experimentally, it is very difficult to conclude whether infinite barriers, as predicted by the VG theory, really exist (distinguishing between infinite or very high barriers becomes unfeasible). Moreover, as commonly observed in other glassy systems, large energy barriers lead to slow relaxations and below a crossover temperature experiments cannot be done in equilibrium.

\section{B. Aging and violation of FDT}

Aging and the modification of the equilibrium FDT are two important properties observed in experiments and simulations of glassy systems and also captured in an analytic solutions to simple mean-field-like models. ${ }^{34,35}$ We here briefly describe them.

\section{Aging}

Consider the two-times global correlation function

$$
C\left(t, t_{w}\right)=\frac{1}{\mathscr{N}}\left\langle O(t) O\left(t_{w}\right)\right\rangle
$$

with $O(t)$ the global observable of interest, and $\mathscr{N}$ a normalization factor ensuring $C(t, t)=1$. In a stationary regime the correlation function depends only on the time difference $\Delta t \equiv t-t_{w}$, i.e. $C\left(t, t_{w}\right)=C(\Delta t)$. Out-of-equilibrium stationarity is not ensured. In relaxing glassy systems it is actually lost. The correlation then depends on both times, $t$ and $t_{w}$, in such a way that the longer the waiting time the slower the decorrelation of the system, i.e. the system is aging. Generally, in the long $t_{w}$ limit, the two-times correlation presents a separation of time scales, with an initial stationary decay for $\Delta t \ll t_{w}$, depending only on $\Delta t$, followed by the aging regime for $\Delta t \gg t_{w}$. This behavior is usually described by

$$
C\left(t, t_{w}\right)=C_{s t}(\Delta t)+C_{a g}\left(t, t_{w}\right),
$$

with $C_{s t}(0)=1-q_{E A}$ and $C_{a g}(t, t)=q_{E A}$, and

$$
\begin{aligned}
\lim _{\Delta t \rightarrow \infty} C_{s t}(\Delta t) & =0, \\
\lim _{\Delta t \rightarrow \infty} \lim _{t_{w} \rightarrow \infty} C\left(t, t_{w}\right) & =q_{E A} .
\end{aligned}
$$

These equations define the Edwards-Anderson order parameter, $q_{E A}$. The correlation function first decays from 1 to $q_{E A}$ in a time-translation invariant manner for $\Delta t \ll t_{w}$. At longer time differences the correlation decays further from $q_{E A}$ to 0 depending on both $t$ and $t_{w}$. This general behavior of the correlation $C$ is sketched in Fig. 2 a).

In many cases the aging part of the correlation can be described as $C_{a g}\left(t, t_{w}\right)=f\left[h(t) / h\left(t_{w}\right)\right]$, with $h(t)$ a system dependent monotonic function. This form is found analytically in simple coarsening systems below their ordering temperature and the $p$-spin disordered models for fragile glasses ${ }^{35}$ It also describes very accurately numerical data for LennardJones mixtures 62 and light-scattering measurements in colloidal suspensions, ${ }^{63}$ among other glass forming particle systems. 
In Fig. 2. b) we present the scaling of the aging part of the correlation shown in Fig. 2] a) using a scaling variable $t / t_{w}$ :

$$
C_{a g}\left(t, t_{w}\right)=\widetilde{C}_{a g}\left(\frac{t}{t_{w}}\right) .
$$

The case $h(t) \propto t^{s}$ with $s$ any power is usually called "simple aging". Note that the short-time stationary regime does not scale in Fig. 2 b). The type of behavior described by (5) is known as additive aging scaling.

Sometimes, the simple aging is slightly modified in favor of a "sub-aging" or "super-aging" scenario in which $h(t)$ is not just a power law of time. In the more common former case the ratio $h(t) / h\left(t_{w}\right)$ can be approximated by $\Delta t / t_{w}^{\mu}$ with $\mu<1$ in the short time-difference limit. These cases are, of course, still compatible with the additive separation in stationary and aging regimes described by (5).

A different, less common, functional form describing the decay of correlations is given by the multiplicative aging scaling:

$$
C\left(t, t_{w}\right)=t_{w}^{-\alpha} \widetilde{C}_{a g}\left(\frac{t}{t_{w}}\right),
$$

where $\alpha>0$ and we have assumed simple aging of the remaining $t$ and $t_{w}$-dependent function. In this case the scaling function is multiplied by a $t_{w}$-dependent correction that eventually makes the aging contribution disappear. The scaling function is such that $\widetilde{C}_{a g}\left(t / t_{w}\right) \sim f(\Delta t) t_{w}^{\alpha}$ for long $t_{w}$ in such a way that the correlation reaches, asymptotically, a stationary regime. The functional form (6) is found in the autocorrelation of the massless scalar field in $d>2,{ }^{64}$ systems at criticality, ${ }^{65.66 .67 .68}$ and a lattice model of a directed polymer in a $1+1$ dimensional random environment ${ }^{69.70 .71 .72}$ In Figs. 2(e) and 2(f) we present a sketch of a correlation function with multiplicative aging and its corresponding scaling form.

Note that the difference between the additive and multiplicative scaling is that in the former the correlation function in double-logarithmic scale has a well-defined plateau at a non-vanishing value separating stationary from aging regimes while in the later the correlation eventually becomes completely stationary and the aging regime disappears.

In a diffusive (or anomalous diffusive) problem, another type of correlation function is preferred, which is simply the displacement

$$
\Delta\left(t, t_{w}\right) \equiv\left\langle\left[O(t)-O\left(t_{w}\right)\right]^{2}\right\rangle,
$$

that can be rewritten in terms of the correlation $C\left(t, t_{w}\right) \equiv$ $\left\langle O(t) O\left(t_{w}\right)\right\rangle$ [note that we are omitting here the normalization factor $\mathscr{N}$ used in [1] as

$$
\Delta\left(t, t_{w}\right)=C(t, t)+C\left(t_{w}, t_{w}\right)-2 C\left(t, t_{w}\right) .
$$

This quantity vanishes at equal times and increases with the time difference. If $C(t, t)$ increases with time and is not bounded then the growth of $\Delta$ is also unbounded. For this type of correlation function it is also possible to write down both the additive and multiplicative aging scaling forms:

$$
\begin{aligned}
& \Delta\left(t, t_{w}\right)=\Delta_{s t}(\Delta t)+\widetilde{\Delta}_{a g}\left(\frac{t}{t_{w}}\right), \\
& \Delta\left(t, t_{w}\right)=t_{w}^{\alpha} \widetilde{\Delta}_{a g}\left(\frac{t}{t_{w}}\right),
\end{aligned}
$$

in the simple aging case (or with a more general function $\mathrm{h}(\mathrm{t})$ appearing in $\Delta_{a g}$ in sub or super aging cases). A typical displacement has initially a ballistic growth, followed by a sub-diffusive regime proportional to $\Delta t^{\alpha}$ with $\alpha<1$, and a normal diffusion regime ( $\alpha=0$ in the additive aging scaling scenario). The displacement of a $D$-dimensional manifold relaxing in an infinite dimensional embedding space after a quench to low-temperature has an additive separation between a stationary and an aging regime as in (9). ${ }^{73.74}$ In the one-dimensional scalar or $N$-component field ${ }^{64}$ and Sinai diffusion ${ }^{75,76,77}$ the displacement follows instead a multiplicative

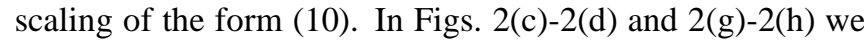
present a sketch of the displacement with additive and multiplicative scaling and their corresponding scaling forms.

\section{The linear response}

In order to measure a linear response the following protocol is commonly used. At $t_{w}$ one applies a perturbation to the system by adding the term $\mathscr{H}_{O}=h O$ of intensity $h$ to the total Hamiltonian. This perturbation is conjugated to the observable $O$. If the perturbation is instantaneous, the linear response is defined as

$$
\left.R\left(t, t_{w}\right) \equiv \frac{\delta\left\langle O_{h}(t)\right\rangle}{\delta h\left(t_{w}\right)}\right|_{h=0} .
$$

If, instead, the perturbation is held applied during the interval $\left[t_{w}, t\right]$ the integrated response associated to the external perturbation and the observable $O$ is

$$
\chi\left(t, t_{w}\right)=\frac{\left\langle O_{h}(t)\right\rangle-\langle O(t)\rangle}{h}
$$

where the sub-index $h$ indicates that $O$ is measured under the field. Similarly to what we explained above for the correlation and displacement, the time-dependence of the integrated linear response can be scaled in an additive or a multiplicative form:

$$
\begin{aligned}
& \chi\left(t, t_{w}\right)=\chi_{s t}(\Delta t)+\chi_{a g}\left(\frac{t}{t_{w}}\right), \\
& \chi\left(t, t_{w}\right)=t_{w}^{\alpha} \widetilde{\chi}_{a g}\left(\frac{t}{t_{w}}\right),
\end{aligned}
$$

respectively, where we assumed simple aging.

\section{The fluctuation-dissipation theorem (FDT)}

In equilibrium the integrated response is stationary, $\chi\left(t, t_{w}\right)=\chi(\Delta t)$, and the fluctuation-dissipation theorem 
(a)

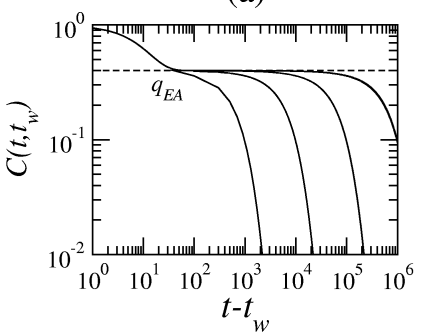

(c)

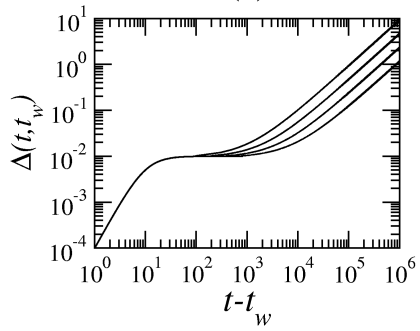

(e)

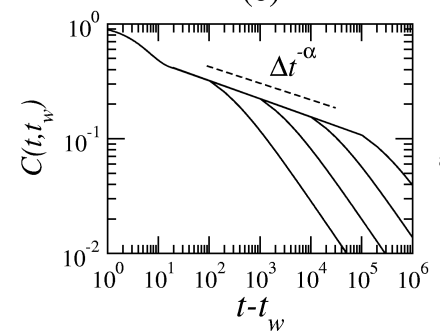

(g)

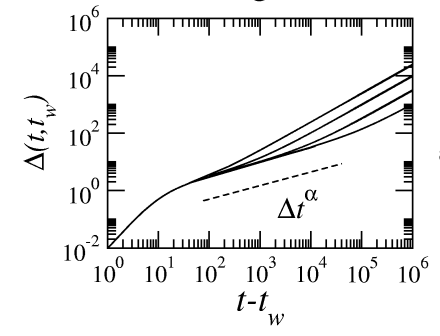

(b)

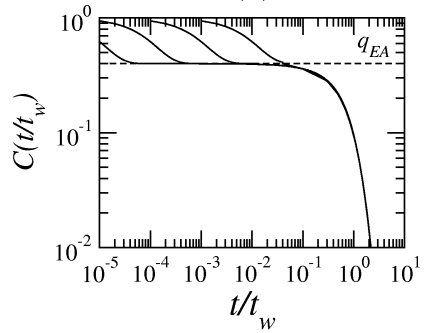

(d)

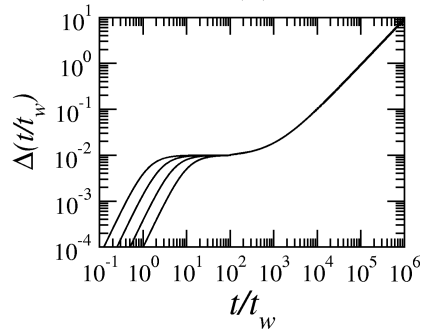

(f)

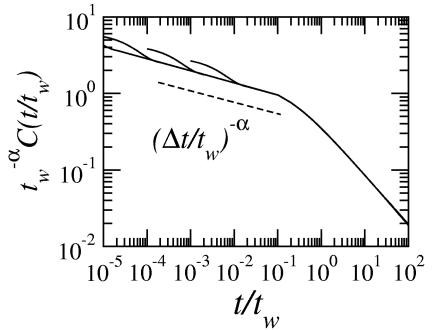

(h)

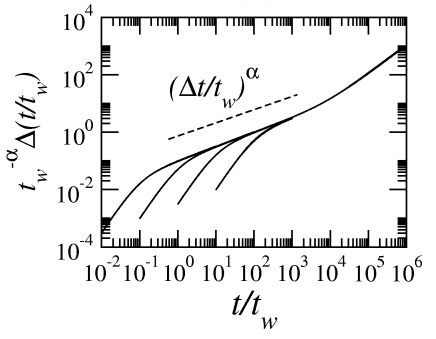

FIG. 2: Sketch of the relaxation of correlations and displacements and their corresponding scaling forms in glassy regimes. (a)-(b) Correlation with additive aging scaling; (c)-(d) displacement with additive scaling; (e)-(f) correlation with multiplicative aging scaling; (g)(h) displacement with multiplicative scaling. Note that the stationary decays at short time-differences in the left panels do not scale in the right panels.

(FDT) implies

$$
\begin{aligned}
\frac{d C(\Delta t)}{d \Delta t} & =-k_{B} T R(\Delta t), \\
C(\Delta t)-C(0) & =-k_{B} T \chi(\Delta t),
\end{aligned}
$$

$(\Delta t \geq 0)$ relating correlation and instantaneous or integrated responses only through the temperature of the environment. In equilibrium the equal-times correlation is constant, and the displacement is simply related to the correlation: $\Delta(\Delta t)=$
$2[1-C(\Delta t)]$. Equations 15 and 16 then imply

$$
\begin{aligned}
\frac{d \Delta(\Delta t)}{d \Delta t} & =2 k_{B} T R(\Delta t), \\
\Delta(\Delta t) & =2 k_{B} T \chi(\Delta t) .
\end{aligned}
$$

In an aging out-of-equilibrium regime the integrated response also depends on two times, see Eqs. (13) and (14). A simple generalization of the FDT (15) reads

$$
\theta\left(t-t_{w}\right) \frac{\partial}{\partial t_{w}} C\left(t, t_{w}\right)=k_{B} T_{\mathrm{eff}}\left(t, t_{w}\right) R\left(t, t_{w}\right),
$$

which gives a definition of a two-times dependent effective temperature $T_{\text {eff }}$. It has been shown that this definition respects the expected properties of a temperature for systems with slow dynamics and a bounded energy 37 Note that the integration of the above relation over a time-interval $\left[t_{w}, t\right]$ can take a very complicated form if $T_{\text {eff }}$ depends on times in an involved manner. If, as it turns out to be in systems with additive scaling, it is piecewise constant one finds a linear relation between the displacement and the integrated linear response in each interval. Systems with multiplicative scaling also allow for the identification of a simple $T_{\text {eff }}$ once the factors $t_{w}^{\alpha}$ are taken into account.

The parametric plots $\chi(C)$ and $\chi(\Delta)$ have become a useful tool to analyze the violation of FDT ${ }^{35,36}$ For systems in equilibrium these plots are just straight lines with slope $-1 / k_{B} T$ and $1 / 2 k_{B} T$, respectively. For glassy systems evolving out of equilibrium one has to distinguish those with an additive from those with a multiplicative scaling. For the former, the parametric plot $\chi(C)[\chi(\Delta)]$ shows a $-1 / k_{B} T\left[1 / 2 k_{B} T\right]$ slope in the quasi-equilibrium short time-difference regime, while at long time-differences - for correlation values below the plateau at $q_{E A}-$ the slope changes to $-1 / k_{B} T^{*}\left[1 / 2 k_{B} T^{*}\right]$. In the asymptotic, $t_{w} \rightarrow \infty$ limit the breaking point in $\chi(C)$ occurs at a fixed point $\left[q_{E A}, \chi_{E A}=1 /\left(k_{B} T\right)\left(1-q_{E A}\right)\right]$. This case is schematically represented in Fig. 3] a). In the case of a system with multiplicative scaling one needs to eliminate the factors $t_{w}^{-\alpha}$ in the decaying correlation ( $t_{w}^{\alpha}$ in the diffusing displacement) and $t_{w}^{\alpha}$ in the integrated response in order to get a stable parametric plot. In other words, tracing $\chi(C)$ $[\chi(\Delta)]$ one gets a similar broken line with slopes $-1 / k_{B} T$ and $-1 / k_{B} T^{*}\left[1 / 2 k_{B} T\right.$ and $\left.1 / 2 k_{B} T^{*}\right]$ but with a breaking point that moves towards $\left(0,1 / k_{B} T\right)[(\infty, \infty)]$ with increasing $t_{w}$. This behaviour is schematically represented in Fig. 3. b). Constructing instead $t_{w}^{-\alpha} \chi\left(t_{w}^{\alpha} C\right)$ or, equivalently, $t_{w}^{-\alpha} \chi\left(t_{w}^{-\alpha} \Delta\right)$ a stable breaking point is obtained and a parametric plot similar to Fig. [3] a) is recovered.

\section{MODEL}

We model vortices in superconductors as a set of elastic lines with an interaction potential $K_{0}(r / \lambda)$ screened at the scale of the London penetration depth $\lambda$. This model was introduced in the theory of flux lattice melting by D. Nelson, ${ }^{78,79}$ it was used to develop the theory of the Bose glass phase,, 80 and it has been used by several authors in the past 

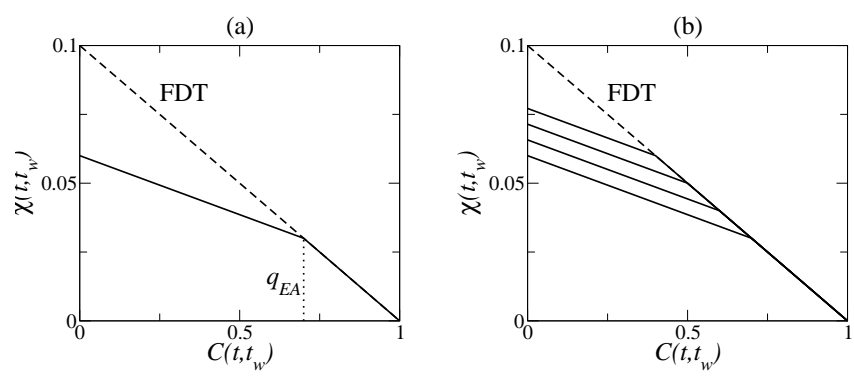

FIG. 3: Sketch of the fluctuation-dissipation relation between integrated response and correlation: (a) additive scaling with a stable breaking point at $q_{E A}$; (b) multiplicative scaling with a breaking point that drifts towards $(0,1 / T)$ in the limit $t_{w} \rightarrow \infty$.

and until present ${ }^{19.81 .82 .83 .84}$ There is now a general consensus in that this model is correct for moderately anisotropic superconductors like YBCO. Numerical simulations find good quantitative agreement with experimental results for this system. 19.81 We have chosen to work with such a well-known model to build upon previously acquired knowledge and addressing questions that have not been considered in the literature yet.

\section{A. Model Hamiltonian}

We consider a model for 3D elastic flux lines in a hightemperature superconductor. The model is composed by $L$ planes labeled with index $z$ and placed a distance $d_{z}$ apart. The direction of the magnetic field $B$ is perpendicular to the planes. The magnetic field fixes the vortex density $n_{B}=B / \Phi_{0}$, where $\Phi_{0}=h c / 2 e$ is the flux quantum perpendicular to the planes. The system contains $N$ flux lines, where the $i$-th flux line is characterized by coordinates $\mathbf{r}_{i}(z)$, which means that each flux line is composed by $L$ elements with two-dimensional inplane coordinates $\mathbf{r}_{i}(z)=\left[x_{i}(z), y_{i}(z)\right]$. These elements are sometimes called "vortex pancakes". 81 The superconductor has anisotropy $\varepsilon=\xi_{c} / \xi_{a b}=\lambda_{a b} / \lambda_{c}$, with $\xi_{a b}$ and $\xi_{c}$ the coherence lengths, and $\lambda_{a b}$ and $\lambda_{c}$ the penetration depths. The axes are such that $c \| z$ and $a b \| \mathbf{r}_{i}$.

Assuming that $\mathbf{r}_{i}(z)$ varies slowly with $z$ the Hamiltonian for a London model of elastic flux lines is $\mathscr{H}=\Sigma_{z} \mathscr{H}_{z}$, with

$$
\mathscr{H}_{z}=\sum_{i} U_{l}\left(\Delta \mathbf{r}_{i z}\right)+U_{d}\left(\mathbf{r}_{i z}\right)+\sum_{i<j} U_{i n}\left(\mathbf{r}_{j z}-\mathbf{r}_{i z}\right),
$$

where $U_{l}, U_{d}$, and $U_{\text {in }}$ are the elastic line energy between two pancakes of the same flux line, the interaction of a flux line with a quenched disorder potential, and the in-plane interaction energy between two different flux lines, respectively. Let us now describe each term in some detail.

The repulsive interaction energy between line elements belonging to different flux lines and in the same plane $z$ is approximated as 19.21 .81

$$
U_{i n}=2 \varepsilon_{0} d_{z} K_{0}\left(r / \lambda_{a b}\right)
$$

where $K_{0}$ is the modified Bessel function, and with

$$
\varepsilon_{0}=\left(\Phi_{0} / 4 \pi \lambda_{a b}\right)^{2}
$$

The elastic line energy of the $i$-th vortex is

$$
U_{l}=\frac{1}{2} c_{l}\left(\partial_{z} \mathbf{r}_{i}\right)^{2} d z
$$

where $c_{l}$ measures the line tension. A natural choice for $d_{z}$ is the distance between $\mathrm{CuO}$ planes, in which case one can account for the effect of the Josephson coupling 81 using

$$
U_{l}=c_{l} \lambda_{J}\left|\partial_{z} \mathbf{r}_{i}\right| \text { for }|\Delta \mathbf{r}|>2 \lambda_{J}
$$

while keeping the previous expression for $|\Delta \mathbf{r}|<2 \lambda_{J}$, where $\lambda_{J}=d_{z} / \varepsilon$ is the Josephson length, and $\Delta \mathbf{r}_{i z}=\mathbf{r}_{i, z+1}-\mathbf{r}_{i, z}$ measures the separation between two adjacent flux line elements.

The quenched disorder potential due to the impurities is 1

$$
U_{d}(\mathbf{r})=\int d^{2} \mathbf{r}^{\prime} u\left(\mathbf{r}^{\prime}\right) p\left(\left|\mathbf{r}-\mathbf{r}^{\prime}\right|\right)
$$

where the form factor is

$$
p(r)=2 \xi_{a b}^{2} /\left(r^{2}+2 \xi_{a b}^{2}\right)
$$

and

$$
\left\langle u(\mathbf{r}, z) u\left(\mathbf{r}^{\prime}, z^{\prime}\right)\right\rangle=\gamma \delta\left(\mathbf{r}-\mathbf{r}^{\prime}\right) \delta_{z z^{\prime}}
$$

defines the disorder strength ${ }^{1,19} \gamma$.

We model the dynamics of the vortex system with the Langevin equation

$$
\eta \frac{\partial \mathbf{r}_{i z}(t)}{\partial t}=-\frac{\delta \mathscr{H}\left[\left\{\mathbf{r}_{i z}(t)\right\}\right]}{\delta \mathbf{r}_{i z}}+\mathbf{f}_{i z}^{T}(t),
$$

where $\eta$ is the Bardeen-Stephen friction coefficient. The thermal force $\mathbf{f}_{i z}^{T}(t)$ satisfies

$$
\left\langle f_{i z, \mu}^{T}(t)\right\rangle=0
$$

and

$$
\left\langle f_{i z, \mu}^{T}(t) f_{i^{\prime} z^{\prime}, \mu^{\prime}}^{T}\left(t^{\prime}\right)\right\rangle=2 \eta k_{B} T \delta\left(t-t^{\prime}\right) \delta_{z z^{\prime}} \delta_{i i^{\prime}} \delta_{\mu \mu^{\prime}}
$$

where $\mu, \mu^{\prime}=x, y$ and $T$ is the thermal bath temperature.

\section{B. Numerical details}

There is no standard numerical procedure to compute the in-plane interaction 21 with periodic boundary conditions. Ryu and Stroud worked with a summation calculation over image vortices, ${ }^{81}$ while Zimányi and coworkers ${ }^{19.21 .85}$ and Nordborg and Blatter ${ }^{82}$ used instead the periodic extension of a simplified Fourier representation. Here we describe the numerical procedure we used to compute the terms contributing to the Hamiltonian 20,

The $z$-planes have dimensions $L_{x} \times L_{y}$ and we used periodic boundary conditions to minimize finite size effects in the 
planes. To compute the in-plane interaction each direction in the $z$-planes is discretized in $N_{x(y)}$ points a distance $a_{x(y)}$ apart, such that $L_{x(y)}=N_{x(y)} a_{x(y)}$ for the $x(y)$-direction. The $K_{0}$ dependence of the interaction potential, Eqs.(19), corresponds to the case of vortices in infinite samples. In a finite sample with periodic boundary conditions, the effective potential that takes into account the effect of the summation over periodic images, $\tilde{K}_{0}(r)$, has to be calculated. Instead of calculating $\tilde{K}_{0}$ in real space, we found more convenient to work in the reciprocal space, using a discrete Fourier transform of the in-plane interaction (21) that accounts for the periodic boundary condition. This reads

$$
\tilde{U}_{i n}=\frac{2 \varepsilon_{0} d_{z} \Phi_{0}}{L_{x} L_{y}} \frac{1}{\lambda_{a b}^{2} h_{k, l}^{2}-1},
$$

with $k$ and $l$ the indices for the discrete reciprocal space, and

$$
h_{k l}^{2}=\frac{2}{a_{x}^{2}}\left[\cos \left(\frac{2 \pi k}{N_{x}}\right)-1\right]+\frac{2}{a_{y}^{2}}\left[\cos \left(\frac{2 \pi l}{N_{y}}\right)-1\right] .
$$

The above expressions account explicitly for the periodic boundary conditions in $L_{x}$ and $L_{y}$. Using The numerical value of $\tilde{U}_{\text {in }}$ is easily computed in a $k l$ discrete mesh. To calculate the real space value of $\tilde{U}_{i n}$, a fast Fourier transform FFTW ${ }^{86}$ routine is used. In Fig. 44 we show the $K_{0}$ and $K_{1}$ Bessel functions corresponding to the infinite system, and the effective functions $\tilde{K}_{0}$ and $\tilde{K}_{1}$ that consider the periodic boundary conditions, obtained with this method. Different values of $\lambda_{a b}$ are shown to follow the general trend. As can be observed, the new functions take into account the periodic boundary conditions: the $\tilde{K}_{1}$ function, which is proportional to the force between vortex pancakes, goes to zero at the edge of the simulation box $\left(L_{x} / 2\right)$, which is a necessary condition when working with periodic boundary conditions. Finally the in-plane force, proportional to $\tilde{K}_{1}\left(r / \lambda_{a b}\right)$, derived from this potential energy is stored in a force table during the computational run.

Since the $z$-direction is discretized in $L$ planes, which naturally correspond to the $\mathrm{CuO}$ planes in a model superconductor, the elastic interaction is discretized as

$$
U_{l}\left(\Delta \mathbf{r}_{i z}\right)= \begin{cases}\frac{1}{2} c_{l}\left(\frac{\Delta \mathbf{r}_{i z}}{d_{z}}\right)^{2} d_{z}, & \text { for }\left|\Delta \mathbf{r}_{i z}\right|>2 \lambda_{J}, \\ c_{l} \lambda_{J} \frac{\left|\Delta \mathbf{r}_{i z}\right|}{d_{z}}, & \text { for }\left|\Delta \mathbf{r}_{i z}\right|>2 \lambda_{J} .\end{cases}
$$

In order to compute the disorder potential 25 we use the same discrete mesh as for the in-plane interaction. The discrete disorder potential at a given mesh point, indexed by $m n$, is given by

$$
U_{d}=2 \sqrt{\frac{24 \sqrt{2} \pi^{2} a_{x} a_{y} \gamma}{d_{z}}} \sum_{m^{\prime}=1}^{N_{x}} \sum_{n^{\prime}=1}^{N_{y}} u_{m^{\prime} n^{\prime}}^{\prime} p_{m^{\prime} n^{\prime}},
$$

where $u_{m n}^{\prime}$ is a random number uniformly distributed between 0 and 1 and thus the prefactors enforce Eq. 27) to hold. The discrete form factor is given by

$$
p_{m n}=\sum_{m^{\prime}=1}^{N_{x}} \sum_{n^{\prime}=1}^{N_{y}}\left[\left(m-m^{\prime}\right)^{2} a_{x}^{2}+\left(n-n^{\prime}\right)^{2} a_{y}^{2}+2\right]^{-1} .
$$

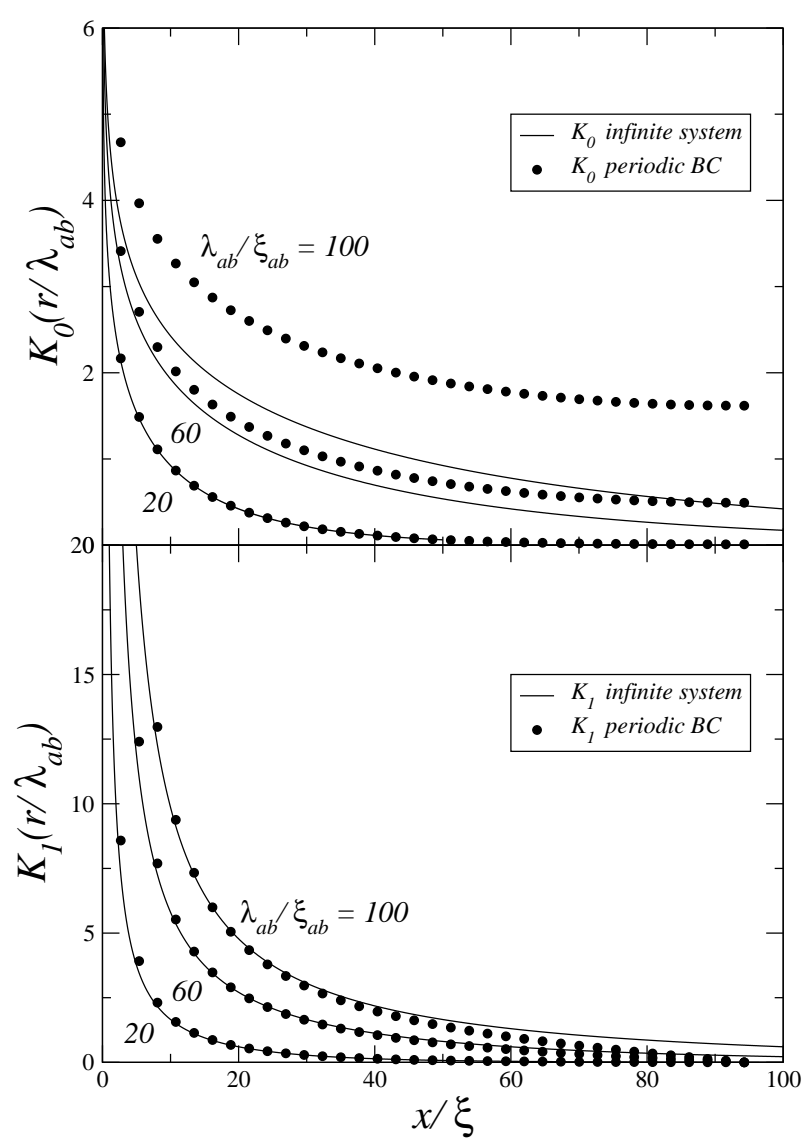

FIG. 4: Comparison between the analytic Bessel functions $K_{0}(x)$ and $K_{1}(x)$ and the numerical results using periodic boundary conditions and $L_{x} / \xi_{a b}=188$. Data is shown up to $x=L_{x} / 2$.

The indices $m$ and $n$ run over the discrete mesh, i.e. $m=$ $1,2, \ldots, N_{x}$ and $n=1,2, \ldots, N_{y}$. In Fig. 5 a typical disorder potential realization is shown for two intensities, $\gamma=10^{-4}$ and $10^{-5}$, later used in the simulations. The force exerted by the disorder potential at a given point of the mesh is stored in a force table.

Since the in-plane and disorder potentials are evaluated in a discrete mesh but the pancake positions are continuous variables in the Langevin dynamics equations, a four-point interpolation method is used.

\section{Parameters of the simulation}

The above model gives a good quantitative description of the vortex physics of moderately anisotropic hightemperature superconductors like $\mathrm{YBCO} \stackrel{1.19 .81 .87 .88}{ }$ We therefore choose the values of the parameters to mimic this system: $\varepsilon=1 / 5, \lambda_{a b} / \xi_{a b}=100, \lambda_{J} / \xi_{a b}=16$, and $c_{l}=$ $\varepsilon^{2} \varepsilon_{0} 2\left[1+\ln \left(\lambda_{a b} / d_{z}\right)\right] / \pi{ }^{81}$ The strength of disorder is set to $\gamma=10^{-5}$, for which case we find that above $B_{c r} \sim 0.002 H_{c 2}$ the system is in the VG at low temperatures (see Section $\nabla$. 


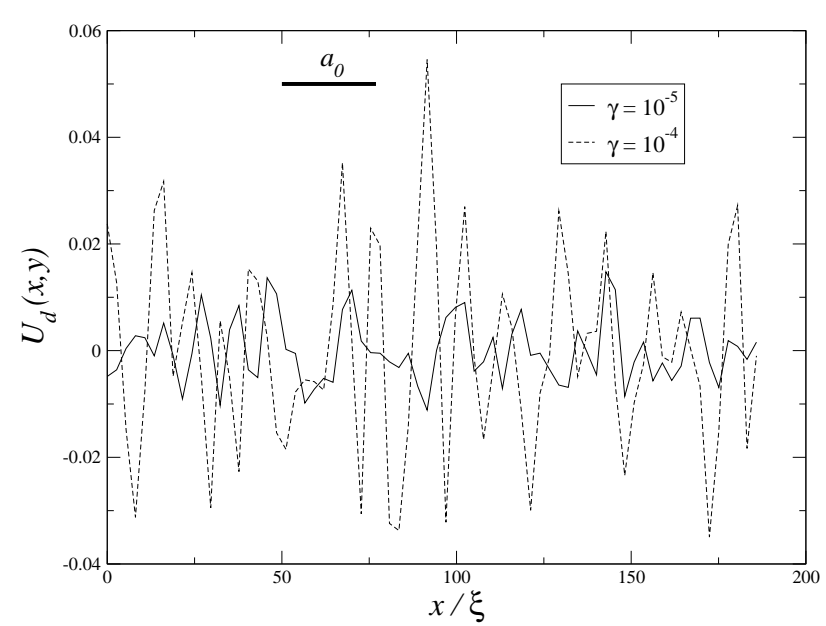

FIG. 5: Quenched disorder potential for two values of the strength $\gamma$. The Abrikosov length $a_{0}=26.935$ is shown for reference.

Time is normalized by $t_{0}=\xi_{a b}^{2} \eta / \varepsilon_{0}$, length by the vortex lattice parameter (or Abrikosov length) $a_{0}=\left[2 \Phi_{0} /(\sqrt{3} B)\right]^{1 / 2}$, energy by $\varepsilon_{0} d_{z}$, and temperature by $\varepsilon_{0} d_{z} / k_{B}$. We simulate $N=56$ vortices in a box of size $7 a_{0} \times 4 \sqrt{3} a_{0}$ with periodic boundary conditions for the in-plane coordinates. The $z$ direction is discretized in $L=50$ planes with free boundary conditions. Averages are performed over 10 realizations of disorder.

\section{QUANTITIES OF INTEREST}

In order to study the out-of-equilibrium dynamics we use a two-times protocol. First the system is equilibrated at an initial high temperature well inside the VL $\left(T_{i}=0.3\right)$ evolving during $t=10^{4}$ steps. Then the system is quenched to a low temperature $T$, where the time count is set to zero. Starting with this far from equilibrium initial condition, the system is let to evolve during a waiting time $t_{w}$, after which the quantities of interest are measured.

With the aim of fully characterizing the dynamical properties of the vortex system we study several $t$ and $t_{w}$ dependent quantities. First we focus on the study of finite size effects by monitoring the roughness of the lines, a quantity that is used in the study of interfaces dynamics, ${ }^{89}$ here generalized to include the $t$ and $t_{w}$ dependence:

$$
\left\langle w^{2}\left(t, t_{w}\right)\right\rangle=\frac{1}{L N} \sum_{i z}\left\langle\left[\delta x_{i z}(t)-\delta x_{i z}\left(t_{w}\right)\right]^{2}\right\rangle,
$$

where $\delta x_{i z}(t)=x_{i z}(t)-\overline{x_{i}(t)}$ accounts for the displacement of the $i z$ line segment relative to the center of mass of the $i$ th elastic line, $\overline{x_{i}(t)}=L^{-1} \sum_{z} x_{i z}(t)$, along one of the two axis of the two-dimensional planes on which the pancakes move. Another quantity that is useful to analyze finite size effects is the dynamic wandering, ${ }^{90} W\left(z, t, t_{w}\right)$, defined as

$$
W\left(z, t, t_{w}\right)=\frac{1}{N} \sum_{i}\left\langle\left[\Delta \mathbf{r}_{i z}(t)-\Delta \mathbf{r}_{i z}\left(t_{w}\right)\right]^{2}\right\rangle,
$$

with $\Delta \mathbf{r}_{i z}(t)=\mathbf{r}_{i z}(t)-\mathbf{r}_{i 0}(t)$. This quantity measures how the displacement of the vortex pancakes (or line elements) in the $z$-th plane correlates with the bottom plane $z=0$ between $t$ and $t_{w}$. Although this was defined for the general vortex model, it is worth mentioning that when in-plane interactions and disorder potential are absent, i.e. for the Edwards-Wilkinson (EW) equation,,$\underline{=1}$ the dynamic wandering is related to the heightheight correlation function studied in surface growth phenomena 89.92 .93 .94

Aging in these systems is best characterized by monitoring the mean-square-displacement (MSD) of the pancakes in the planes

$$
B\left(t, t_{w}\right)=\frac{1}{L N} \sum_{i z}\left\langle\left[x_{i z}(t)-x_{i z}\left(t_{w}\right)\right]^{2}\right\rangle,
$$

and the two-times wave-vector dependent density-density correlation function

$$
C_{k}\left(t, t_{w}\right)=\frac{1}{L N} \sum_{i z}\left\langle e^{-i k\left[x_{i z}(t)-x_{i z}\left(t_{w}\right)\right]}\right\rangle
$$

that has been analysed in great detail in relaxing glass forming liquids ${ }^{62}$ The mean-square displacement $B$ is of the form of the generic displacements $\Delta$ discussed and the density-density correlation is of the form of the generic decaying correlation $C$ discussed in Sec.IIIB

In order to complete the characterization of the aging properties and to study the modifications of the FDT in the out-ofequilibrium regime, we measure a linear response function by applying a random force of the form $\mathbf{f}_{i z}=\delta s_{i z} \hat{\mathbf{x}}$ at time $t_{w}$ on a replica of the system, where $\delta$ is the intensity of the perturbation, and $s_{i z}= \pm 1$ with equal probability ${ }^{38.95}$ The integrated response is

$$
\chi\left(t, t_{w}\right)=\frac{1}{L N \delta} \sum_{l z}\left\langle s_{i z}\left[x_{i z}^{\delta}(t)-x_{i z}(t)\right]\right\rangle,
$$

where $x_{i z}^{\delta}$ and $x_{i z}$ correspond to the position evaluated in two replicas of the system, with and without the perturbation. The equilibrium FDT implies

$$
B(\Delta t)=2 k_{B} T \chi(\Delta t),
$$

where the $\Delta t=t-t_{w}$ argument represents stationarity. In the aging regime the FDT is violated and one constructs the modified relation

$$
B\left(t, t_{w}\right)=2 k_{B} T_{\mathrm{eff}}\left(t, t_{w}\right) \chi\left(t, t_{w}\right) .
$$

\section{VORTEX GLASS}

The vortex phase diagram of a model very similar to the one studied in this work was obtained by Zimányi and co-workers,$\frac{19}{19}$ and it has the qualitative behavior shown in Fig. 21b). In order to show that we are in the VG region of the phase diagram for the present model superconductor, we study the behavior of the in-plane vortex structure factor and the behavior of dynamic correlations and relaxation times in the VL. 


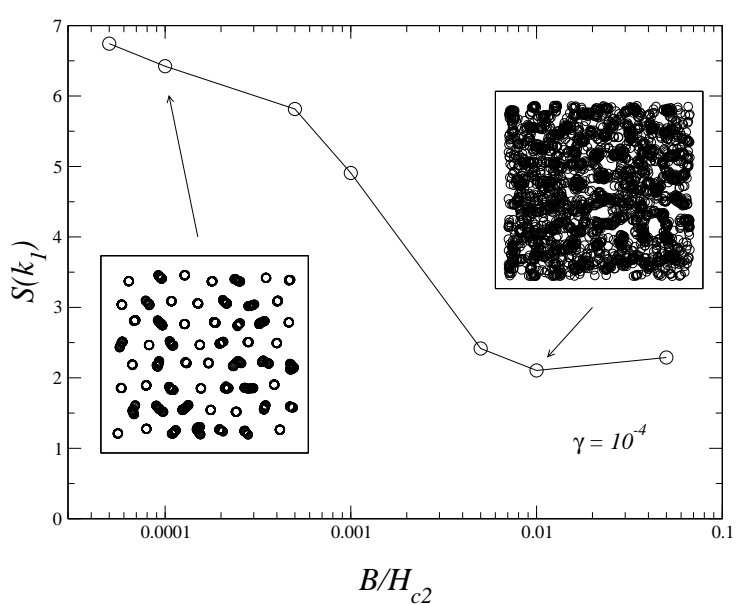

FIG. 6: Structure factor at $k_{1}$, the main peak of the Abrikosov lattice, and for the disorder intensity $\gamma=10^{-5}$ as a function of the vortex density parametrized by the field $B$. Insets: a number of superposed snapshots of the system.

\section{A. Structure factor: loss of crystalline order}

In Fig. 6 the vortex structure factor at $k_{1}$, the principal peak of the Abrikosov lattice with parameter $a_{0}$, is shown while varying the magnetic field intensity $B$ (proportional to the vortex density) and at fixed disorder intensity $\gamma=10^{-5}$. For this case we find that above $B_{c r} \sim 0.002 H_{c 2}$ the Bragg peaks disappear and the flux lines are frozen in a highly amorphous structure at low temperatures. Furthermore, $B_{c r}$ is dependent on the disorder intensity $\gamma$. We therefore choose to study the case with $B=0.01 H_{c 2} \gg B_{c r}$, which is deep within the $\mathrm{VG}$ regime at low $T$ for both $\gamma=10^{-4}$ and $10^{-5}$. The region with higher structure factor $S\left(k_{1}\right)$ below $B_{c r}$ presumably corresponds to a BG phase, but to be sure one should test the finite size behavior of the spatial correlations and structure factor. The insets clearly show the structural differences between two snapshots with different densities. These snapshots are projections of the two-dimensional pancake coordinates of the $L$ planes into the $z=0$ plane.

\section{B. Diffusion in the VL and dynamical arrest}

Once working at fields $B>B_{c r}$, upon increasing temperature the system should go through the VG-VL crossover line. This could be numerically observed as a sudden increase of the vortex diffusion. However, since the "pancake" diffusion is sub-linear ${ }^{81}$ in the VL the data analysis and the identification of the crossover line may be subtle. This can be better analyzed starting at high temperatures, from the VL side, and studying the relaxation of dynamical correlation functions

In order to clarify the nature of the dynamic arrest and to define a crossover glass temperature $T_{g}$, we therefore study the change in diffusion upon decreasing the temperature in the VL phase, by analyzing the MSD defined in Eq. 38.

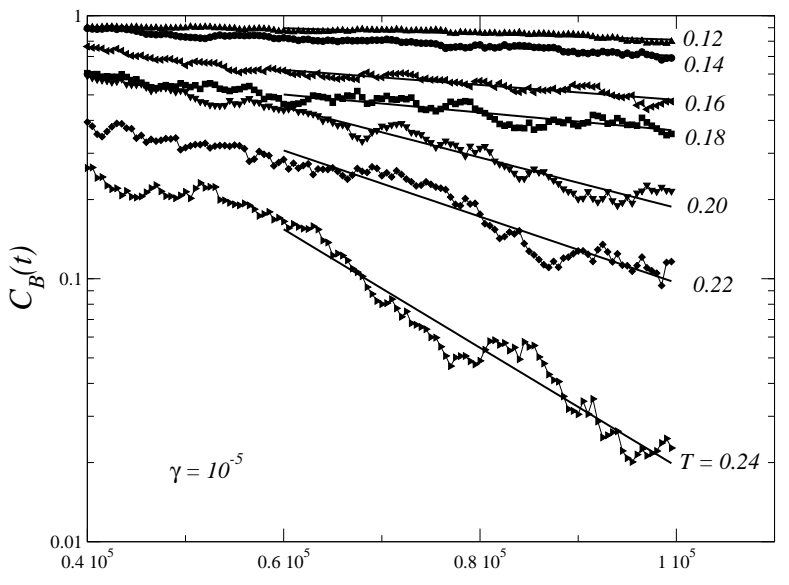

FIG. 7: Correlation $C_{B}(t)$ for different temperatures and $\gamma=10^{-5}$. Each curve is labeled by the temperature. The corresponding exponential fits are shown.

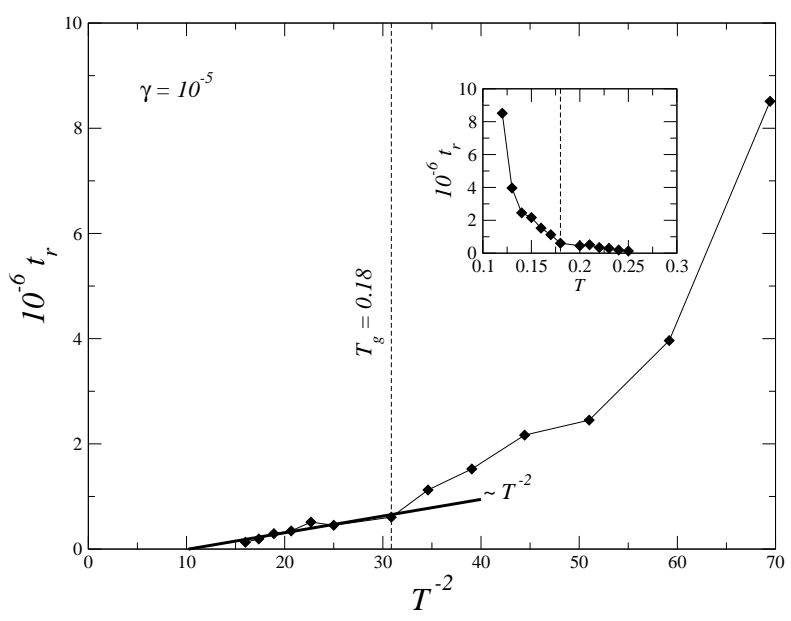

FIG. 8: Relaxation times $t_{r}$ obtained from the exponential fit $C_{B}(t)=$ $A e^{-t / t_{r}}$. The crossover temperature $T_{g}$, where the VL relaxation time departs from the single line behavior $t_{r} \propto 1 / T^{2}$ is quoted. The inset shows the sudden increase in $t_{r}$ with decreasing temperature.

At high temperatures, well inside the VL, the system can be easily equilibrated and the MSD depends on $\Delta t=t-t_{w}$, $B\left(t, t_{w}\right) \equiv B(\Delta t)$. Two regimes of pancake diffusion can be observed in this case. For times longer than a saturation time, $\Delta t>t_{x}$, diffusion is normal, $B(\Delta t) \sim \Delta t$, corresponding to the diffusion of the center of mass of each line. At shorter times, $\Delta t<t_{x}$, the diffusion of the pancakes corresponds to that of line segments in a line in which the longitudinal correlation length is still growing. This is the same as considering an infinite line $L \rightarrow \infty$ and it is characterized by sub-diffusion,, 81 $B(\Delta t) \sim \Delta t^{1 / 2}$.

One aims to extract a characteristic relaxation time from the diffusion evolution. To this end, Zimányi and coworkers ${ }^{19}$ used an exponential fit, $C(t)=A e^{-t / t_{r}}$, to describe the corre- 
lation

$$
C(t)=\exp \left\{-\overline{\left.\langle\mathbf{r}(t)-\langle\mathbf{r}(t)\rangle]^{2}\right\rangle}\right\}
$$

where $\mathbf{r}(t)$ measures the two-dimensional in-plane displacements of the flux line elements. However, this is only consistent with a normal diffusion regime, and should be carefully interpreted. In their simulations they used an equilibration time of $10^{5}$ and measured $C(t)$ for the following $10^{5}$ time interval. This is the same as using a waiting time of $10^{5}$ from the given initial condition. The exponential fit is valid if $t_{x}<10^{5}$, corresponding to center of mass diffusion.

As mentioned above, Ryu and Stroud ${ }^{81}$ studied the melting of the VL using a different model and observed that the pancake diffusion is sub-linear, $B(\Delta t) \sim \Delta t^{\alpha}$ with the exponent $\alpha=1 / 2$ at high temperatures and $\alpha<1 / 2$ at low temperatures. This is consistent with our results.

Within the same scheme, we calculate the evolution of the correlation for the largest waiting time used in our simulations, $t_{w}=10^{5}$,

$$
C_{B}(t)=\exp \left\{-\left[B\left(t, t_{w}=10^{5}\right)\right]^{2}\right\},
$$

and fit it to the form $C_{B}(t)=A e^{-t / t_{r}}$, that follows after using the sub-linear exponent $\alpha=1 / 2$. In Fig. [7we show this correlation in a log-linear representation for different temperatures and $\gamma=10^{-5}$. The fits for $\Delta t>0.6 \times 10^{5}$ are also shown. From this data we obtain the relaxation time $t_{r}$ presented in Fig. 8 The inset shows that there is a sudden increase in the relaxation time upon decreasing temperature. For noninteracting flux lines the temperature dependence of the relaxation time should be $t_{r} \propto 1 / T^{2}, 1,81$ and the same is expected deep in the VL. The main panel in Fig. 8 shows that indeed for high temperatures $t_{r}$ grows linearly with $1 / T^{2}$. Below a temperature $T_{g} \approx 0.18$ we observe that the relaxation time $t_{r}$ departs strongly from the $1 / T^{2}$-dependence and presents a remarkable growth. Furthermore, for $T<T_{g}$ is not possible to equilibrate the system and correlation functions depend on the two times, $t$ and $t_{w}$, as we will analyze in Sec.VIII Therefore, the temperature $T_{g}$ signals the crossover from the equilibrium high-temperature regime to the out-of-equilibrium lowtemperature regime.

\section{FINITE SIZE EFFECTS}

Before analyzing the out-of-equilibrium behavior of different correlation and response functions of the VG, it is necessary to previously identify finite size effects in the dynamics of the system. We here compare finite size effects in the single elastic line dynamics with those in the much more complex systems we are dealing with, a set of interacting lines in the presence of quenched disorder. To this end we study both the two-times roughness (36) and the dynamic wandering 37. We also show how finite size effects appear in two other quantities of interest, the pancake MSD (38) and the densitydensity correlation function [39].

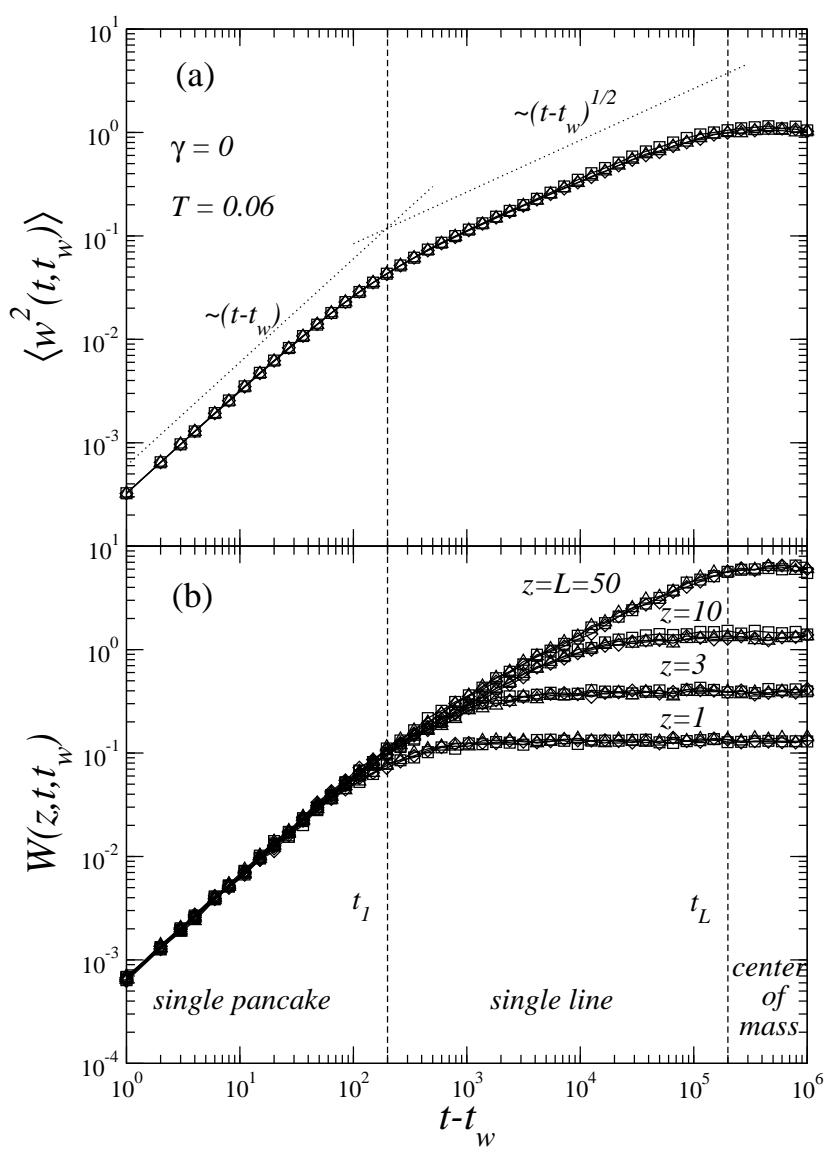

FIG. 9: (a) Roughness and (b) dynamic wandering of noninteracting elastic lines without quenched disorder (EW elastic lines) at $T=0.06$. Three dynamic regimes are highlighted, corresponding to single pancake, single line, and center of mass dynamics. Note that different curves with $t_{w}=10^{4}, 10^{5}$ and $10^{6}$ (open squares, open diamonds and open triangles, respectively) overlap, showing that these quantities are in a stationary regime.

\section{A. Single lines: the Edwards-Wilkinson equation}

When the in-plane interactions and disorder potential are absent from the Hamiltonian (20), Eq. (28) reduces to $N$ decoupled Edwards-Wilkinson (EW) equations ${ }^{91}$ The EW equation was studied in great detail in the context of growing interface dynamics ${ }^{89}$ After a transient the roughness of the EW interface becomes stationary and it follows the FamilyVicsek scaling ${ }^{96}$

$$
\left\langle w^{2}(\Delta t)\right\rangle=L^{2 \zeta} f\left(\frac{\Delta t}{t_{x}}\right)
$$

where $t_{x} \sim L^{\theta}$ is the saturation time, $L$ is the system size, and $f(x)$ is a scaling function with $f(x) \sim x^{2 \beta}$ for $x \ll 1$ and $f(x) \sim$ cons. for $x \gg 1$. The growth and roughness exponents, $\beta$ and $\zeta$ respectively, are related to the dynamic exponent $\theta$ through the scaling relation $\theta=\zeta / \beta$. The exponents take values $\zeta=1 / 2, \beta=1 / 4$, and $\theta=2$. This scaling form clearly 


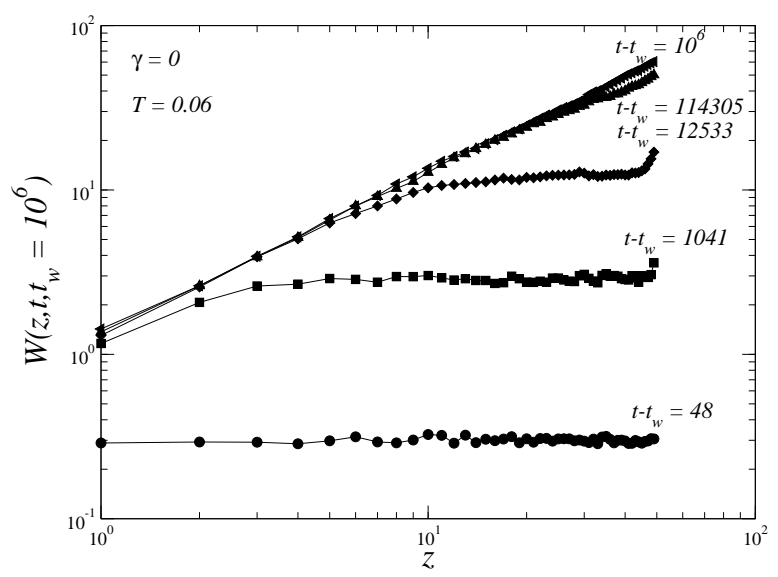

FIG. 10: Dynamic wandering as a function of the plane index $z$. $T=0.06, L=50$ each curve is labeled by $\Delta t$ and the waiting time is $t_{w}=10^{6}$ for all. Since $W(z, \Delta t)$ saturates at a value proportional to the longitudinal correlation length $\xi_{\|}$, the growth of $\xi_{\|}$is observed for increasing $\Delta t$ at fixed $z$.

states how the system size is involved in the line dynamics. Other quantities, such as the global correlation function, can however be non-stationary and show aging 64

In order to check how the size and time dependence of our elastic lines compare to the EW ones, we set disorder and in-plane interactions to zero and we analyse the full $\Delta t$ dependence of the roughness and dynamic wandering once the stationary regime has been reached. In Fig. 9 a) we show the time-evolution of the roughness. Three regimes are clear in the figure. For $\Delta t<t_{1}$ the roughness grows as $\left\langle w^{2}\right\rangle \sim \Delta t$ which corresponds to a regime without elastic interactions between the line segments. For $\Delta t>t_{L}$ the roughness saturates to an $L$ dependent value, while for $t_{1}<\Delta t<t_{L}$ the roughness grows as $\left\langle w^{2}\right\rangle \sim \Delta t^{1 / 2}$. The last two regimes are the ones in the scaling function of the Family-Vicsek scaling (45), where $t_{x} \equiv t_{L}$.

To better understand the meaning of a characteristic distance-dependent time $t_{z}$ let us analyze the behavior of the dynamic wandering in Fig. 9 b). For $\Delta t<t_{1}$ the dynamic wandering is independent of $z$ and grows as $\sim \Delta t$. This is the regime in which the line segment in one plane evolves independently of the line segment in any other plane, i.e. there is no truly elastic interaction yet. For $\Delta t>t_{1}$ the line segments belonging to two adjacent planes are elastically interacting and their relative position saturates to a given value. However, at this time, line segments separated by more than a single plane are still uncorrelated. This corresponds to the growth of a longitudinal correlation length, $\xi_{\|}$, along the $z$-direction. This correlation grows as $\xi_{\|}(\Delta t) \sim \Delta t^{1 / \theta}$ for $\Delta t \ll t_{x}$ and saturates at the system size, ${ }^{89} \xi_{\|}(\Delta t) \sim L$, for $\Delta t \gg t_{x}$. The time $t_{z}$ signals the moment when the correlation length is of the order of $z$, i.e. $\xi_{\|}\left(t_{z}\right) \sim z$. In Fig. 9 b) the behavior of $W\left(z, t, t_{w}\right)$ is presented for $z=1,3,10$ and $z=L=50$, where it is shown that the dynamic wandering is essentially a measure of the longitudinal correlation length (the short time $\Delta t^{1 / 2}$ regime

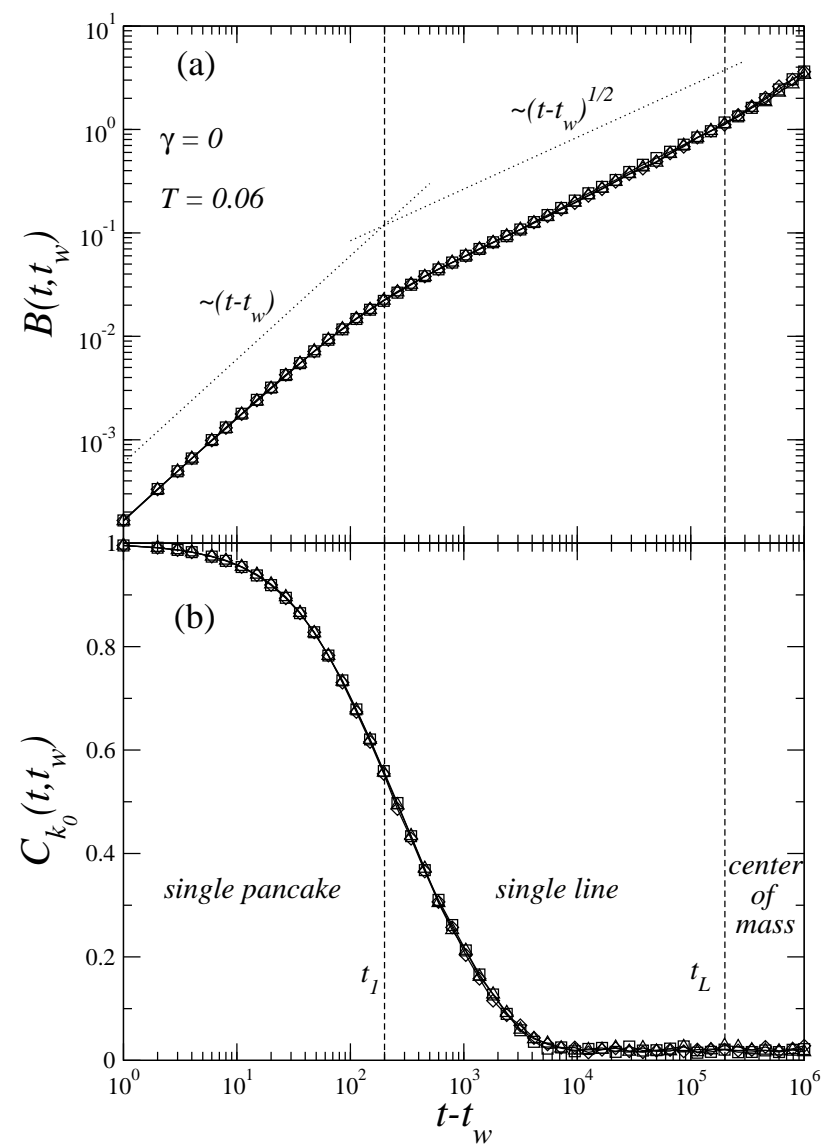

FIG. 11: (a) Pancake MSD $B$ and (b) correlation $C_{k_{0}}$ for the same parameters as in Figs. 9 and 10 $k_{0}$ is the wave vector at the first peak of the structure factor. The three dynamic regimes are clear in $B$, but the correlation $C_{k_{0}}$ does not present any signature of them. Symbols are as in Fig. 9

is not completely developed since the system size is not big enough).

In Fig. 10 we show the $z$-dependence of the dynamic wandering. Since there is no $t_{w}$-dependence, we show here different curves labeled by the corresponding $\Delta t$ value for a single waiting time $t_{w}=10^{6}$. For $\Delta t<t_{1}$, the dynamic wandering does not depend on $z$ since line segments are independent of each other. For $t_{1}<\Delta t<t_{x}$ two regimes are observed. For $z<\xi_{\|}(\Delta t)$ one has $W \sim z$, while for $z>\xi_{\|}(\Delta t)$ the dynamic wandering saturates at a $\Delta t$-dependent value. The final growth near $z=L$ is due to the free boundary conditions of the line. Finally, for $\Delta t>t_{x}$ all the line is correlated and $W \sim z$ for all $z$.

The growth of the longitudinal correlation length is also clear in other quantities. For instance, in Fig. 11 a) we show the pancake MSD $B\left(t, t_{w}\right)$, where the three dynamic regimes are also observed. For $\Delta t<t_{1}$ independent pancake diffusion is observed and diffusion is normal, $B(\Delta t) \sim \Delta t$. For $t_{1}<\Delta t<$ $t_{x}$ diffusion is characterized by a sub-linear law $B(\Delta t) \sim \Delta t^{1 / 2}$, corresponding to the single line regime where every pancake 


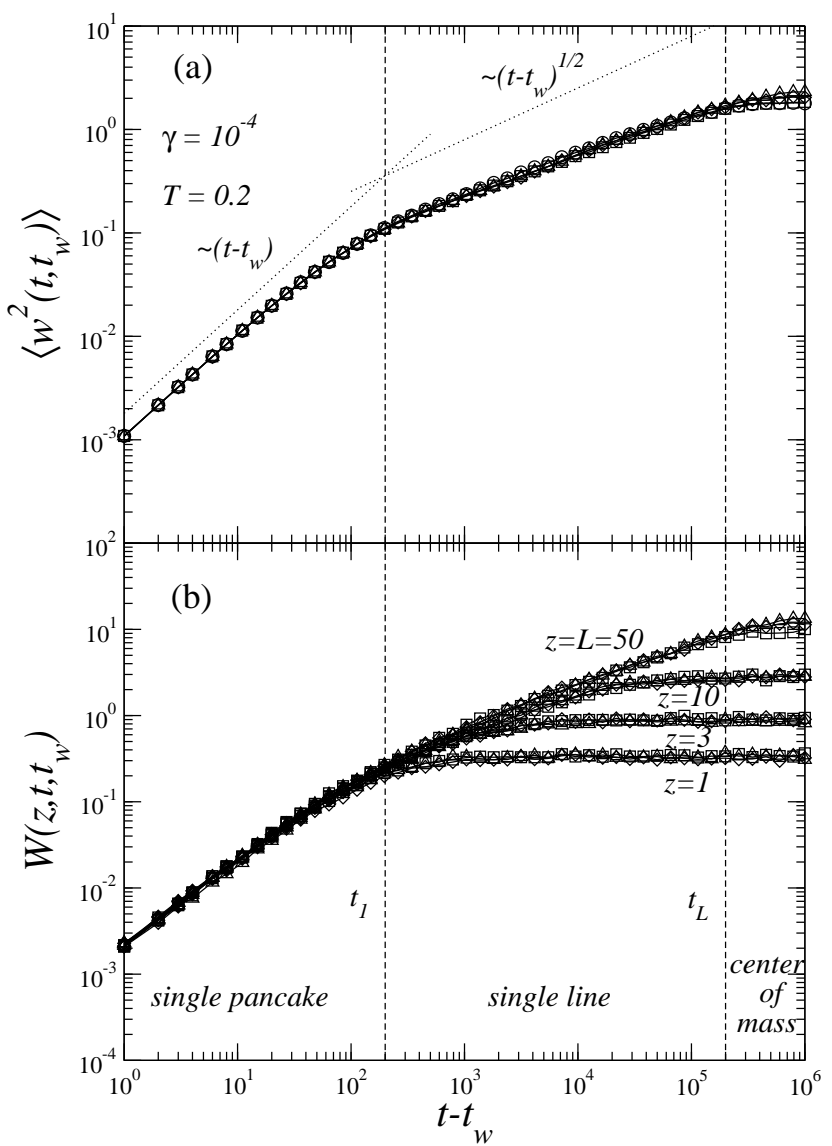

FIG. 12: (a) Roughness and (b) dynamic wandering in the VL at $T=0.2$ with disorder intensity $\gamma=10^{-4}$ (cfr. Fig. 9 where the same measurements for the EW line are shown).

elastically feels the others $\frac{1.81}{2}$ Finally, for $\Delta t>t_{x}$, when the correlation length is of the order of the system size, a trend to normal diffusion corresponding to the center of mass diffusion is observed.

Lastly, in Fig. 11 b) the density-density correlation function $C_{k_{0}}\left(t, t_{w}\right)$ is shown, where $k_{0}$ is the position of the first maximum of the static structure factor. This correlation shows how the position of the pancakes decorrelate in time. Note that around $\Delta t \sim 10^{4}$ the pancakes have lost all information about the position at $t_{w}(\Delta t=0)$ and $C_{k_{0}}$ has effectively decayed to zero. There is no signature of the three dynamic regimes in the decay of the correlation function.

In this subsection we demonstrated how the system size is involved in different dynamical quantities. We also identified three main dynamic regimes. In the following subsections we study how these features are reflected in the VL and VG.

\section{B. The vortex liquid}

Here we return to the case with in-plane interactions and disorder, and present results at high temperature, $T=0.2$, and

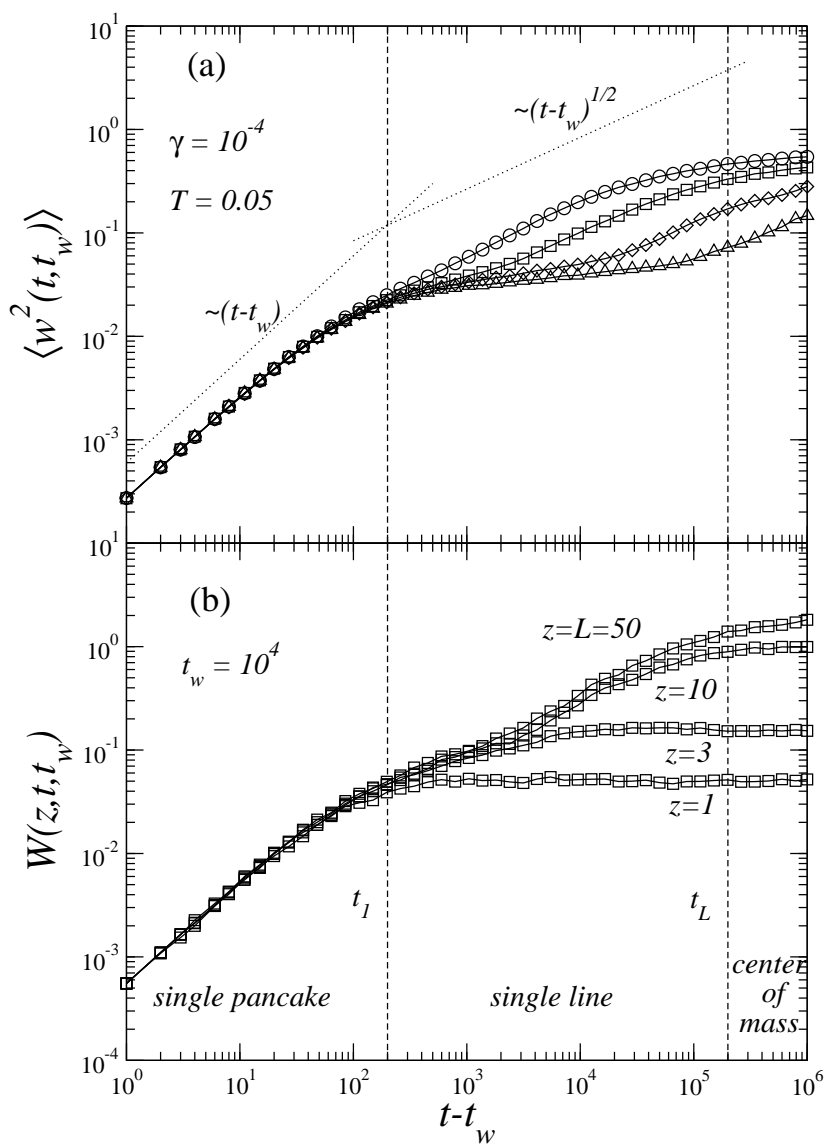

FIG. 13: (a) Roughness and (b) dynamic wandering in the VG at $T=0.05$ with disorder intensity $\gamma=10^{-4}$ (cfr. Figs. 9 and 12 where the same measurements in the EW line and the VL, respectively, are shown). In (b) the dynamic wandering is shown only for $t_{w}=10^{4}$.

with disorder intensity $\gamma=10^{-4}$, well inside the VL. The analysis of finite size effects in the roughness and dynamic wandering (Fig. 12) shows that these are similar to the ones in the EW line. The values of $t_{z}$ are of the same order. At this high temperature and for this disorder intensity one can argue that thermal fluctuations are greater than disorder induced fluctuations, and the disorder potential in (20) can be basically neglected. Moreover, although in-plane interactions are present, at the working vortex density they do not much affect the dynamics leading to essentially the same finite size effects as for the EW line. At higher densities in-plane interactions should become the most relevant interaction of the problem. (As an example, a change in the scaling exponent was found with hard-core line interactions ${ }^{97}$ )

\section{The vortex glass}

Here we analyze how the finite size effects reflect in the low temperature phase. We show data for $T=0.05$ and $\gamma=10^{-4}$. We anticipate that aging effects appear at low temperature, but 


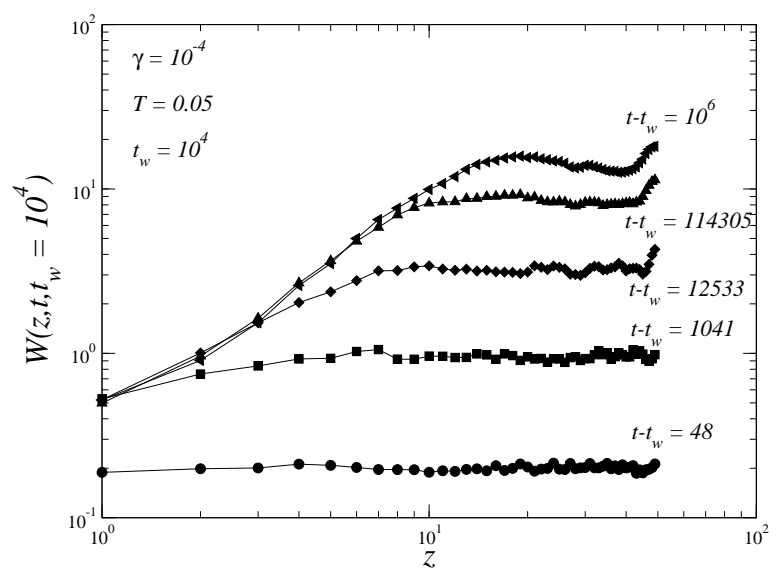

FIG. 14: Dynamic wandering as a function of the plane index $z$. At this low temperature and for this disorder strength the system is aging. We show data for $t_{w}=10^{4}$ and each curve is labeled by $\Delta t$. A change in the slope of $W$ at short $z$ is apparent. For this value of $t_{w}$ the longitudinal correlation length $\xi_{\|}\left(\Delta t=10^{6}\right)$ does not reach the system size $L$.

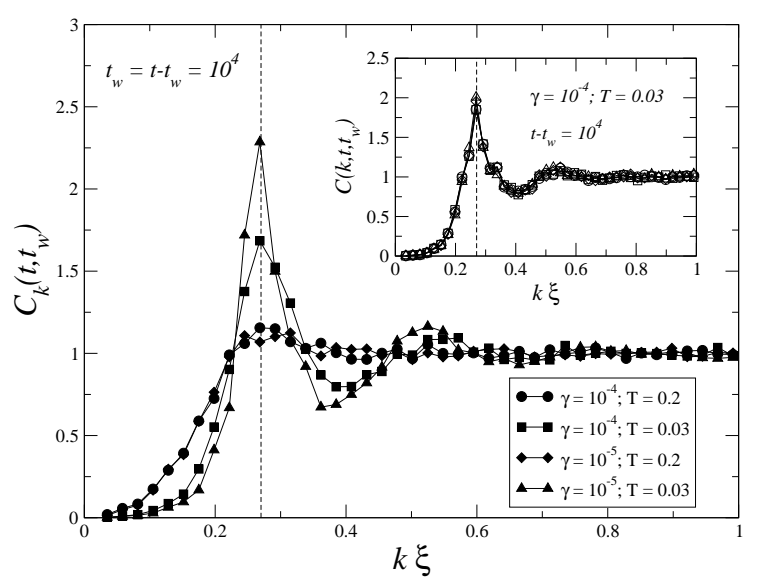

FIG. 15: Normalized static structure factor $C_{k}\left(t, t_{w}\right)$ for $\Delta t=t_{w}=$ $10^{4}$ and different $T$ and $\gamma$ given in the key. The value $k_{0}=0.27$ corresponds to the first peak. The inset shows $C_{k}\left(t, t_{w}\right)$ for $\Delta t=10^{4}$ and different waiting-times, $t_{w}=10,10^{2}, 10^{3}$ and $10^{4}$, with $\gamma=10^{-4}$ and $T=0.03$. The structure factor does not vary significantly with waiting time while the system is aging.

\section{AGING IN THE VORTEX GLASS}

In this Section we describe aging features observed in the present simulations. As shown in Sec. $\nabla \mathrm{B}$ when entering the VG phase, for $T<T_{g}$, is not possible to equilibrate the system, and correlation and response functions depend on the two times, $t$ and $t_{w}$, showing aging. We start by describing the $t_{w}$ and $k$-dependence of the correlation $C_{k}\left(t, t_{w}\right)$. Then we discuss the aging characteristics of the pancake MSD $B\left(t, t_{w}\right)$ and its associated integrated linear response $\chi\left(t, t_{w}\right)$.

A. $C_{k}\left(t, t_{w}\right)$

time is apparent. In Fig. 14 the $z$-dependence of the dynamic wandering for $t_{w}=10^{4}$ is shown. Note that for this value of $t_{w}$ and the maximum $\Delta t=10^{6}$ reached, the longitudinal correlation length does not reach the system size $L$, and then the $\Delta t=10^{6}$ curve shows a saturation regime for high $z$. Although the same trend as in the EW case is present, a difference is observed in the $\xi_{\|}(\Delta t)<z$ regime. For instance, the curves with $\Delta t=114305$ and $\Delta t=10^{6}$ show a change in the slope. This is related to the fact that the roughness exponent $\zeta$ of the FamilyVicsek scaling (45) changes with disorder ${ }^{89.98}$ In the presence of disorder, at a given temperature there exists a characteristic length $z_{c}(T)$ separating two values of $\zeta$. For $z<z_{c}(T)$ one has $\zeta=\zeta_{T}$, while for $z>z_{c}(T)$ one has $\zeta=\zeta_{D}$, with $\zeta_{T}<\zeta_{D}$, where $\zeta_{T}$ and $\zeta_{D}$ are the thermal (EW) and disorder scaling exponents, respectively.

From the above considerations we choose to work in the time regime corresponding to both $\Delta t$ and $t_{w}$ being shorter than $t_{x}$. This ensures that aging features do not overlap saturation effects and finite size effects should be irrelevant. For $L=50$ this corresponds to times $\Delta t, t_{w} \leq 10^{4}$.
Let us first present the $k$-dependence of the normalized static structure factor $C_{k}\left(t, t_{w}\right)$ for fixed $\Delta t$ and $t_{w}$. In Fig.15 we present data for $\Delta t=t_{w}=10^{4}$ and different $T$ and $\gamma$ given in the key. At low temperature, in the VG phase, the curves present a well defined first maximum, followed by an observable second peak, but clearly not showing the presence of an ordered structure. At high temperature, well in the VL phase, the first peak is not as sharp as in the VG case, but it is clearly observed. For reference, the first peak corresponds to $k_{0}=0.27$. This is the value that we used in Fig. 111 In the inset of Fig. 15] the evolution of $C_{k}\left(t, t_{w}\right)$ for fixed $\Delta t$ with waiting time is shown. Data correspond to $\gamma=10^{-4}$ and $T=0.03$. The structure factor is not strongly modified while the system is aging, i.e. while the relaxation time increases for increasing $t_{w}$ (see Fig. 16 below). This feature is also observed in simulations of structural glass formers using Lennard-Jones potentials ${ }^{62}$

Figure 16 shows the evolution of the correlation $C_{k_{0}}\left(t, t_{w}\right)$ at two temperatures, $T=0.2>T_{g}$ and $T=0.03<T_{g}$, and for 


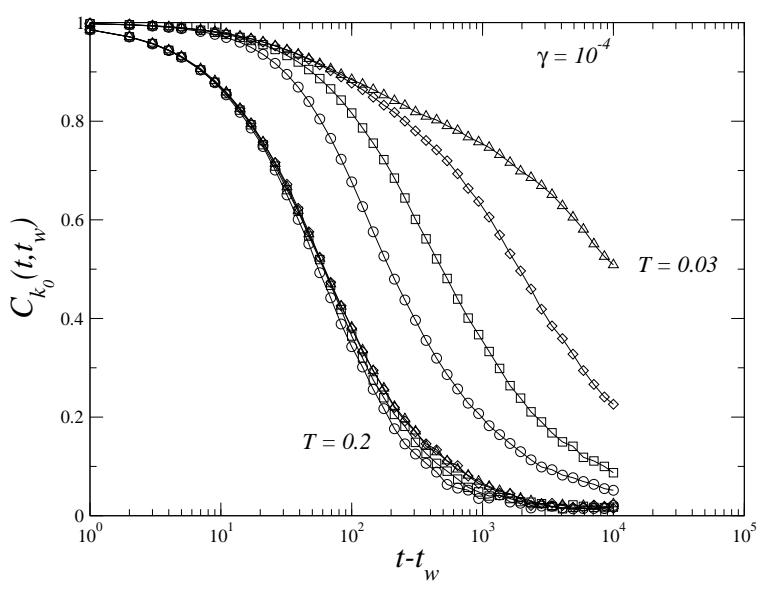

FIG. 16: Correlation $C_{k_{0}}\left(t, t_{w}\right)$ for $\gamma=10^{-4}$ and two temperatures, $T=0.2$ and $T=0.03$. Different waiting times are shown: circles, squares, diamonds and triangles correspond to $t_{w}=10,10^{2}, 10^{3}$ and $10^{4}$, respectively.

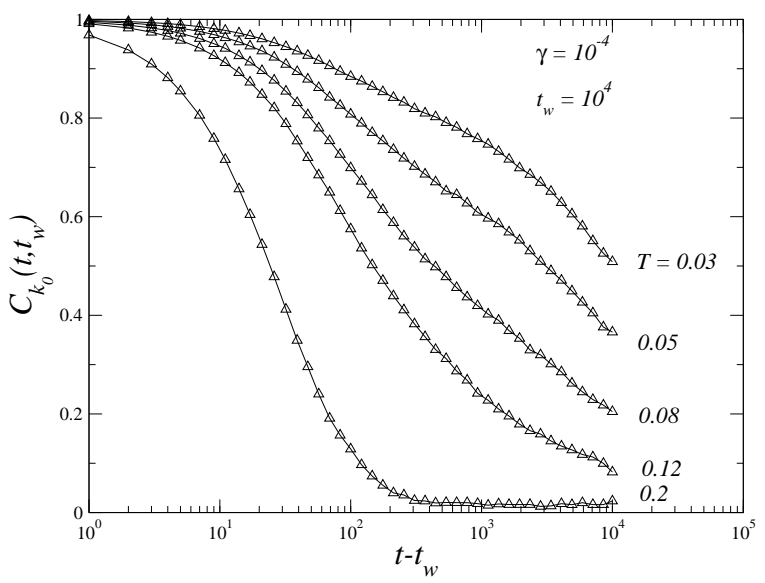

FIG. 17: Correlation $C_{k_{0}}\left(t, t_{w}\right)$ for fixed $t_{w}=10^{4}$ and $\gamma=10^{-4}$. Different curves correspond to different temperatures given in the figure.

different waiting times. The disorder intensity is $\gamma=10^{-4}$. At high temperature the system decorrelates rapidly and the curves become stationary, i.e. they do not depend on $t_{w}$. At low temperature a short time-difference quasi-equilibrium regime is present, but a clear $t_{w}$-dependence is developed at longer time-differences. The system decorrelates slower at longer waiting times, i.e. the system ages. For the longer $t_{w}$ value the curve does not clearly present a plateau as it usually does in other glassy systems ${ }^{36}$ An additive scaling scenario would require that the plateau develops at a longer $t_{w}$. To exclude this possibility we should reach a longer $t_{w}$ while still being in the $t_{w}<t_{x}$ regime. This demands to use larger system sizes $L$. We believe, however, that this is not necessary since the correlation actually decays according to a multiplicative

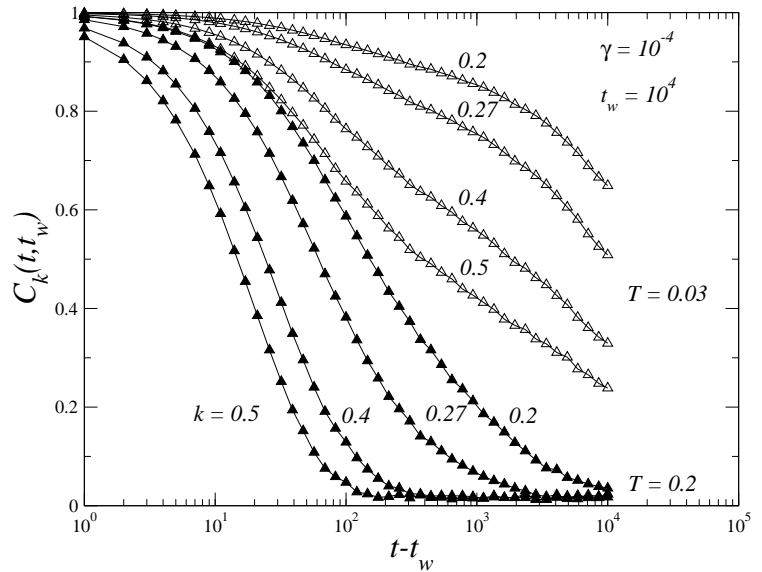

FIG. 18: Correlation $C_{k}\left(t, t_{w}\right)$ for fixed $t_{w}=10^{4}$ and two temperatures, $T=0.2$ and $T=0.03$ with $\gamma=10^{-4}$. Curves for different wave-vector values $k$ are shown.

scaling. We give further support to this proposal below.

The temperature dependence of $C_{k_{0}}\left(t, t_{w}\right)$ for $t_{w}=10^{4}$ is shown in Fig. 17 At the highest temperature the system is in equilibrium but for the remaining values it evolves out of equilibrium. The relaxation time decreases for decreasing temperature (at fixed $t_{w}$ ).

Figure 18 shows the $k$-dependence of $C_{k}\left(t, t_{w}\right)$ for $t_{w}=10^{4}$ and two temperatures, $T=0.2$ and $T=0.03$. At both temperatures the system decorrelates faster with decreasing wavevector $k$, meaning that the relaxation is much faster on small than on large length scales. This is exactly the same behavior observed in the Lennard-Jones glass-forming liquid out of equilibrium by Kob and Barrat. ${ }^{62}$

\section{B. $B\left(t, t_{w}\right)$}

In Fig. 19 the time and temperature evolution of the pancake $\operatorname{MSD}^{50} B\left(t, t_{w}\right)$ is shown for different waiting times and fixed disorder intensity $\gamma=10^{-5} . B\left(t, t_{w}\right)$ is stationary for the high temperature value $T=0.2$. For temperatures lower than $T_{g} \approx 0.18$ the aging behavior develops. As temperature is decreased the separation between curves with different waiting times increases, indicating that the system is deeper in the aging regime. Figure 20 shows the temperature dependence of $B\left(t, t_{w}\right)$ for two waiting times, $t_{w}=10^{2}$ in Fig. 20 a) and $t_{w}=10^{4}$ in Fig. 20 b). After the initial single pancake regime, $B \sim \Delta t$, the sub-diffusive elastic line regime develops and $B \sim \Delta t^{\alpha}$. In the VL $\alpha=1 / 2$, while below some glass crossover temperature to be determined below, $\alpha(T)<1 / 2$. The scaling properties of $B\left(t, t_{w}\right)$ will be analyzed in Section VIII The same behavior is observed using $\gamma=10^{-4}$. 


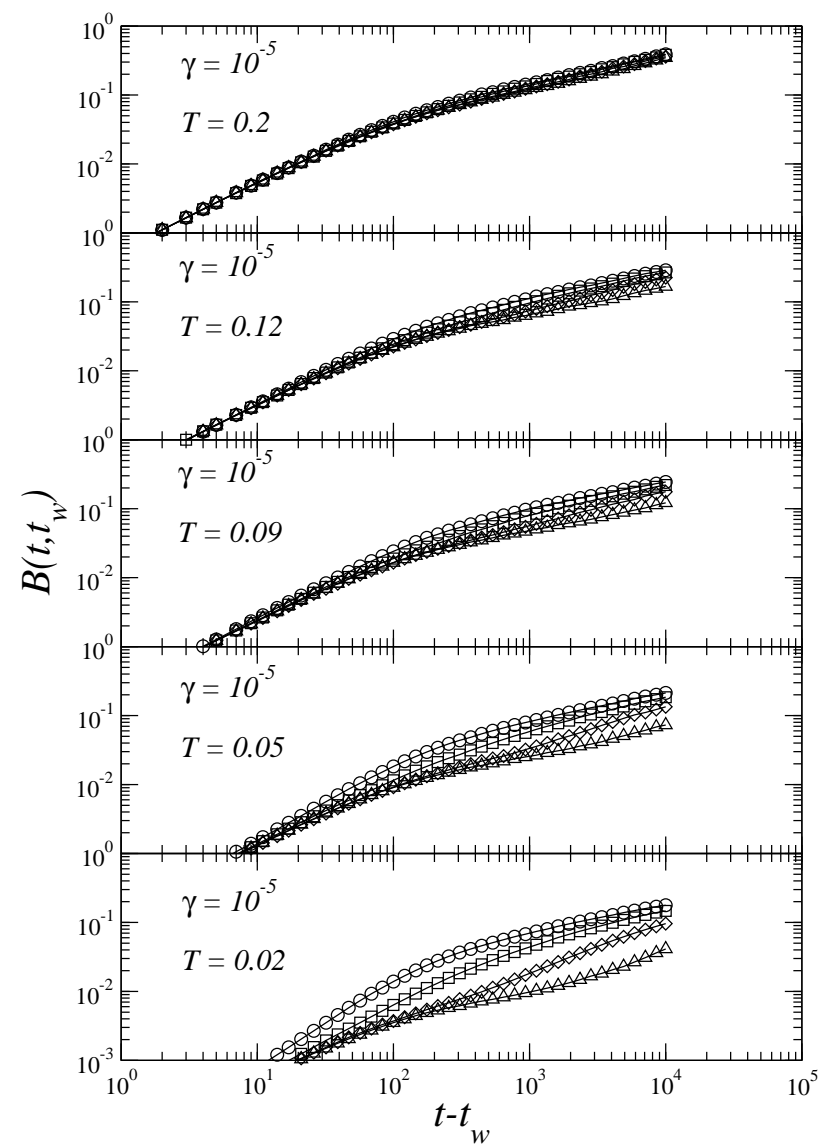

FIG. 19: Pancake MSD $B\left(t, t_{w}\right)$ at different temperatures. Circles, squares, diamonds and triangles correspond to $t_{w}=10,10^{2}, 10^{3}$ and $10^{4}$, respectively.

\section{C. $\chi\left(t, t_{w}\right)$}

In Fig. 21] the dependence of the response function $\chi\left(t, t_{w}\right)$ with $t$ and $t_{w}$ is shown for $\gamma=10^{-5}$ and $T=0.02<T_{g}$. There is also an aging behavior, but not as severe as that of $B\left(t, t_{w}\right)$ for the same parameters (Fig. 19 lower panel). This fact is related to the type of scaling associated to the aging regime, which will be analyzed in Sec.VIII

\section{Comparison with aging of the single elastic line}

We present here results for the out-of-equilibrium dynamics of single elastic lines with disorder, i.e. without the in-plane vortex-vortex interaction. In Figs. 22 and 23 we present the evolution with time of $B\left(t, t_{w}\right)$ and $C\left(t, t_{w}\right)$ for the high and low temperature regimes, $T=0.2$ and $T=0.05$, respectively. At high temperature the dynamics is stationary, not showing any $t_{w}$-dependence. At low temperature the system ages, as observed in the $t_{w}$-dependence of both correlations in Fig.23 These features were also observed in the related model of the directed polymer in random media, $\stackrel{71,90}{ }$ and are qualitatively

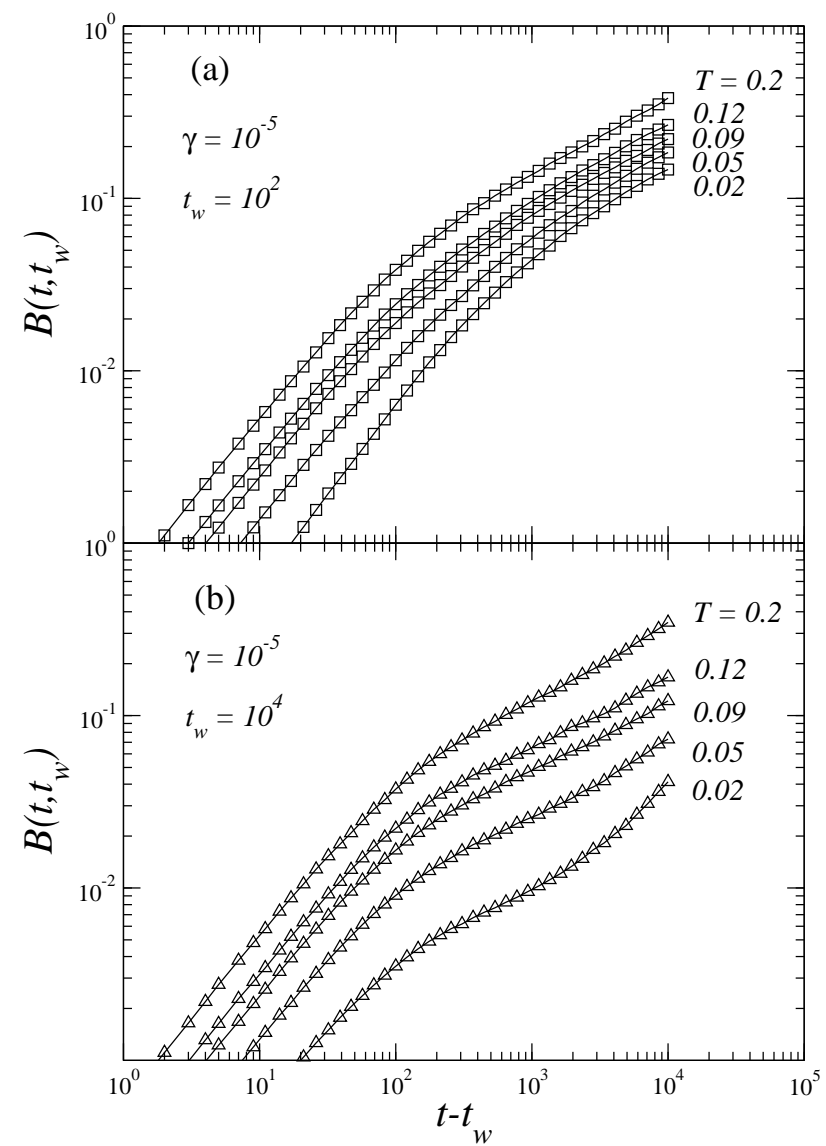

FIG. 20: $B\left(t, t_{w}\right)$ for different temperatures and for two waiting times: (a) $t_{w}=10^{2}$ and (b) $t_{w}=10^{4}$.

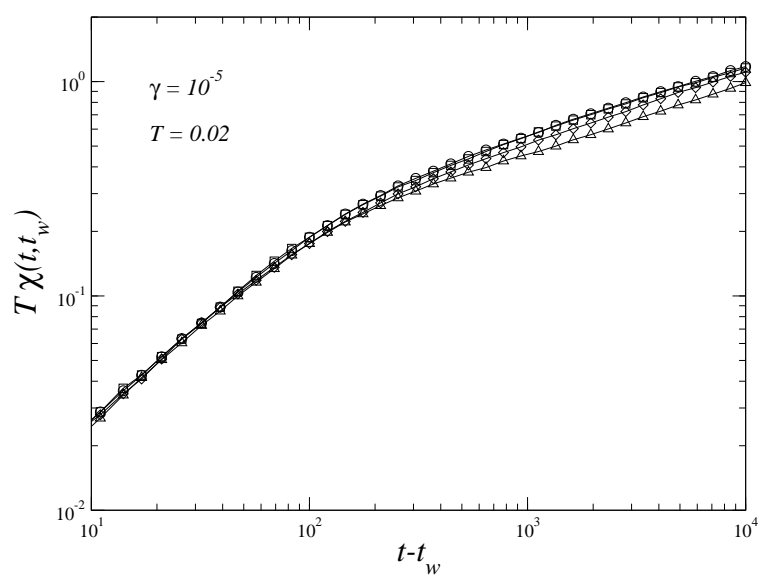

FIG. 21: Integrated response $T \chi\left(t, t_{w}\right)$, where we include the temperature prefactor $\left(k_{B}=1\right)$. The data correspond to $\gamma=10^{-5}$ and $T=0.02$. 


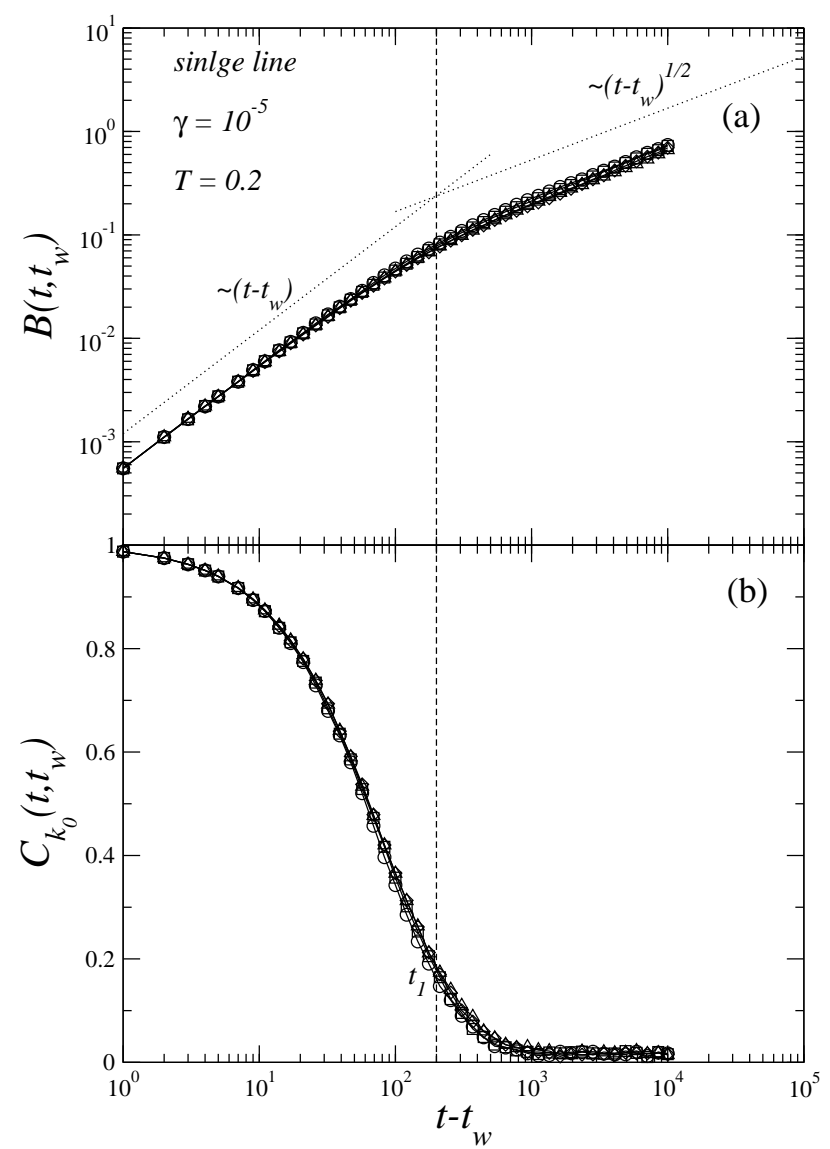

FIG. 22: Stationary dynamics in the high- $T$ regime $(T=0.2)$ of the single line, i.e. without in-plane interactions. (a) $B\left(t, t_{w}\right)$ and (b) $C_{k_{0}}\left(t, t_{w}\right)$ for $\gamma=10^{-5}$ and different waiting times. The data for $t_{w}=10^{3}, 10^{4}, 10^{5}$, and $10^{6}$ collapse on a master curve.

the same as those presented in Sect.VIfor the full interacting VG. This suggests that the out-of-equilibrium dynamics of the interacting vortex problem is dominated by the relaxation of the elastic line. We return to this important issue when analyzing the FDT violation in the next Section.

\section{AGING SCALING AND FDT VIOLATION}

\section{A. Multiplicative aging}

In this Section we study the two-times scaling of $B\left(t, t_{w}\right)$ and $\chi\left(t, t_{w}\right)$ in the aging regime as well as the violation of the FDT and the definition of the effective temperature.

In Fig. 24 we show different scaling scenarii for $B\left(t, t_{w}\right)$ at $T=0.02$. On one hand, in Fig. 24 a) an additive sub-aging scaling is put to the test by plotting $B\left(t, t_{w}\right)$ vs. $\left(t-t_{w}\right) / t_{w}^{\mu}$, while the unscaled $B\left(t, t_{w}\right)$ data is shown in the inset. On the other hand, in Fig. 24 b) the multiplicative scaling of Eq. (6) is used by plotting $\tilde{B}=t_{w}^{\alpha} \cdot B\left(t, t_{w}\right)$ vs. $\left(t-t_{w}\right) / t_{w}$. Although the sub-aging scaling with a low $\mu$ exponent seems appro-

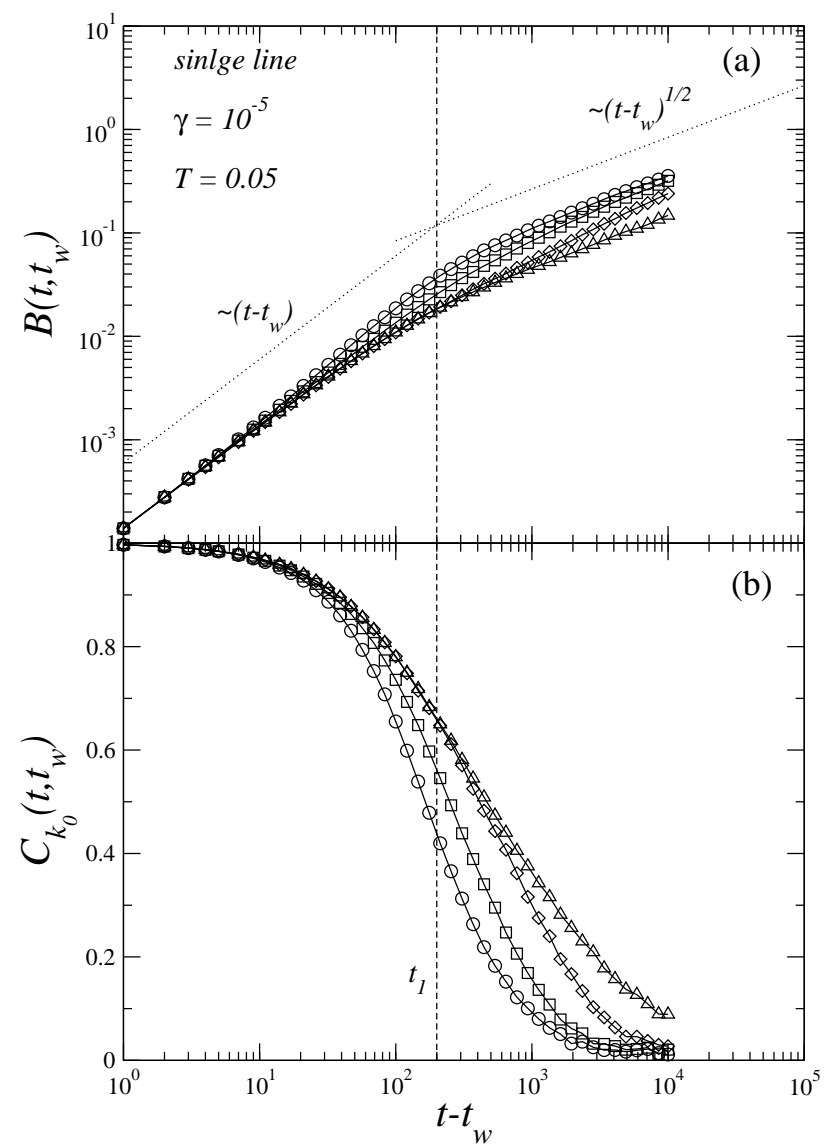

FIG. 23: Aging in the low- $T$ regime $(T=0.05)$ of the single line case, i.e. without in-plane interactions. (a) $B\left(t, t_{w}\right)$ and (b) $C_{k_{0}}\left(t, t_{w}\right)$ for $\gamma=10^{-5}$ and different waiting times: $t_{w}=10^{3}, 10^{4}, 10^{5}$ and $10^{6}$ from left to right.

priate for long time-differences we prefer the multiplicative scaling for at least two important reasons. First, the bare data shown in the inset to panel (a) do not show a plateau at a constant $B$ nor any clear trend to develop it at longer $t_{w}$ 's. This tends to disqualify the additive scaling for which a plateau is expected. Second, the multiplicative scaling allows for a better data collapse that includes the short time-difference sub-diffusive regime, only leaving apart the very short timedifference regime with normal diffusion. Thus, we find that our data is very satisfactorily described within a multiplicative aging scaling scenario. This scaling is similar to the one proposed for the low temperature behavior of the directed polymer in random media ${ }^{71}$ and Sinai's diffusion ${ }^{75,76,77}$ Following Yoshino ${ }^{71}$ we tried the scaling form

$$
B\left(t, t_{w}\right)=t_{w}^{\alpha} \tilde{B}(\tilde{t})
$$

$$
2 k_{B} T \chi\left(t, t_{w}\right)=t_{w}^{\alpha} \tilde{\chi}(\tilde{t}),
$$



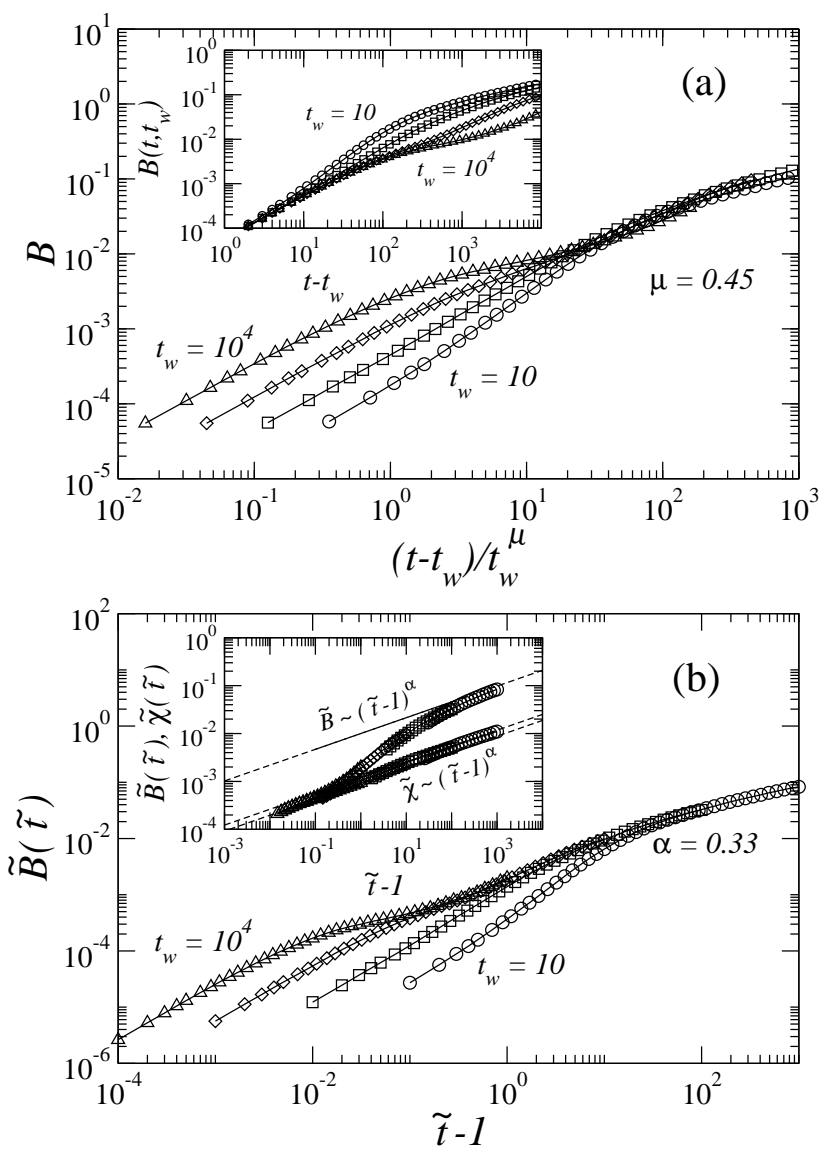

FIG. 24: (a) Test of a sub-aging additive scaling for the pancake MSD for $\gamma=10^{-5}$ and $T=0.02$. The bare data are included in the inset. (b) Multiplicative scaling for the same data as in (a). The values of the scaling exponents $\mu$ and $\alpha$ are quoted. A better data collapse is observed in the last case, including the short time regime. In the inset of (b), the scaled MSD and the integrated response function are shown. Different waiting times collapse on the same master curve. The violation of the FDT at long scaled times $\tilde{t} \gg 1$ is clear.

with $\tilde{t}=t / t_{w}$ and the scaled variables $\tilde{B}$ and $\tilde{\chi}$ given by

$$
\begin{aligned}
& \tilde{B}(\tilde{t})= \begin{cases}c_{1}(T)(\tilde{t}-1)^{\alpha(T)} & \tilde{t} \ll 1, \\
c_{2}(T)(\tilde{t}-1)^{\alpha(T)} & \tilde{t} \gg 1,\end{cases} \\
& \tilde{\chi}(\tilde{t})= \begin{cases}c_{1}(T)(\tilde{t}-1)^{\alpha(T)} & \tilde{t} \ll 1, \\
y(T) c_{2}(T)(\tilde{t}-1)^{\alpha(T)} & \tilde{t} \gg 1,\end{cases}
\end{aligned}
$$

where $c_{1}, c_{2}$ and $y$ are temperature-dependent coefficients (see the discussion in Sect. VIIIB). We assumed a linear dependence of $\tilde{\chi}$ with $\tilde{B}$, but a non-trivial function $\tilde{\chi}(\tilde{B})$, as found in the scalar field or Edwards-Wilkinson equation in $D=2$ and the spin-wave approximation to the 2D XY model,, 4.65 cannot be discarded.

In the inset to Fig. 24 b) the scaled $\tilde{B}$ and $\tilde{\chi}$ at $T=0.02$ are shown. The data for different $t_{w}$ fall on two master curves. $\tilde{B}$ and $\tilde{\chi}$ coincide for $\tilde{t} \ll 1$, which means that FDT holds, while

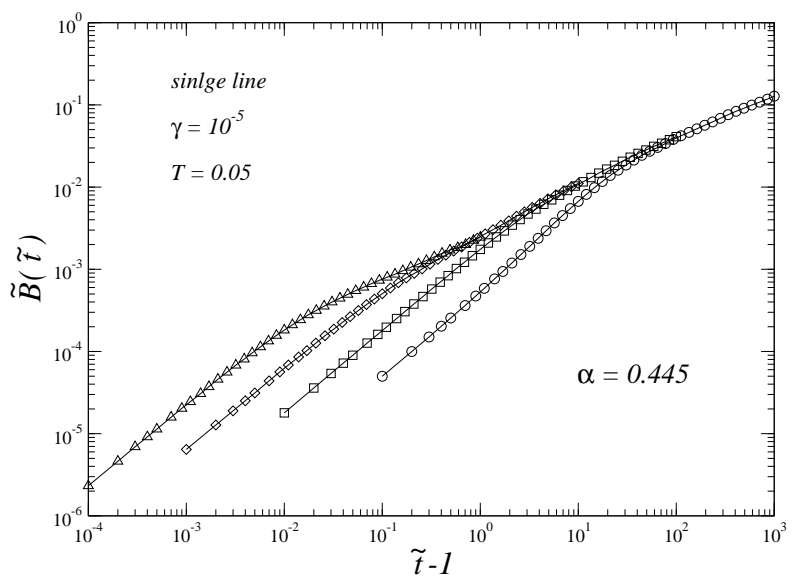

FIG. 25: A single elastic line in a disordered environment: multiplicative scaling of $B\left(t, t_{w}\right)$ for the data shown in Fig[23 a). $\gamma=10^{-5}$ and $T=0.05$. The value of the scaling exponent $\alpha$ is given in the key. Data for different waiting times collapse, except for the stationary normal diffusion regime at very short time-differences.

for longer times $\tilde{t} \gg 1$, the value $y(T)<1$ signals a violation of FDT, which we will analyze in Sec.VIIIC

In Fig.25 the multiplicative scaling for the single line without in-plane interactions is shown. The curves correspond to $\gamma=10^{-5}, T=0.05$ and different waiting times. Besides showing that this type of scaling is appropriate, this figure emphasizes that the only regime not satisfying the scaling is the very short stationary diffusion regime, as suggested from the general picture in Fig. 2

\section{B. Growing length}

Scaling arguments suggest that the averaged dynamics of an elastic line in a random environment should be determined by a single growing length scale, that separates equilibration at short length-scales from non-equilibrium at long lengthscales. This is also, essentially, the picture that dictates dynamic scaling in coarsening systems.

The multiplicative scaling of Eqs. (46a) and (46b) implictly assume a growing length with a power-law dependence in time. However, the analytic determination of such a timedependent length is very hard; it is known in a small number of problems such as ferromagnetic domain growth in clean systems with conserved and non-conserved order parameter ${ }^{99}$ But even these presumably simple cases can be plagued with pre-asymptotic regimes, as shown for instance by Krząkała for the 2D Ising model with Kawasaki dynamics 100

In the case of an elastic string in a random environment a phenomenological creep argument complemented with the assumption that typical barriers scale as $L^{\theta}$ leads to

$$
L(t) \sim L_{c}\left[\frac{T}{U_{c}} \ln \left(\frac{t}{t_{0}}\right)\right]^{1 / \theta}
$$


asymptotically, i.e. in the limit of very long times and very large scales. $L_{c}$ is the Larkin length, $U_{c}$ its corresponding energy scale and $t_{0}$ a microscopic time scale. The exponent $\theta$ is usually further assumed to take the same value as for the free-energy fluctuations, $\theta=1 / 3$. Alternatively, a similar argument with logarithmically growing barriers imply a power law growth of the typical length scale $L(t)$.

Yoshino ${ }^{70.90}$ and Kolton et a l. $^{101}$ studied the growing length-scale numerically in a lattice and a continuous model of a single line in a random environment, respectively. Both studies show that $L(t)$ crosses over from a power-law to a logarithmic growth at a typical time-scale $t^{*}$ associated to a typical length scale $L^{*}$. For instance, Kolton et al found $t^{*} \sim 10^{4}$ and $L^{*} \sim 80$ using lines with total lengths $L=256$ and $L=512$. Similar values were found by Yoshino.

In our study of vortices in interaction we were forced to use rather short total lengths, $L=50$. Hence, we may suppose our dynamics falls in the first regime in which $L(t)$ grows as a power law. Indeed, all our data can be satisfactorily scaled in a manner that is consistent with such a power law. We may expect, clearly, that the crossover in the growing length will induce a change in the scaling forms (46a) and 46b) possibly to a more general form

$$
\begin{aligned}
& B\left(t, t_{w}\right) \sim L^{\zeta}\left(t_{w}\right) \widetilde{B}_{a g}\left(\frac{L(t)}{L\left(t_{w}\right)}\right), \\
& \chi\left(t, t_{w}\right) \sim L^{\bar{\zeta}}\left(t_{w}\right) \widetilde{\chi}_{a g}\left(\frac{L(t)}{L\left(t_{w}\right)}\right) .
\end{aligned}
$$

For times $t<t^{*}$, with a behavior $L(t) \sim t^{\alpha / \zeta}$ and $\bar{\zeta}=\zeta$ one recovers Eqs. (46a) and 46b. For longer times a growing $L(t)$ as in Eq. (48) is also possible. Testing the latest time-regime, however, goes beyond the scope of this study. The value of the exponent $\bar{\zeta}$ is not completely clear yet. Our numerical data support $\bar{\zeta}=\zeta$ but at longer times one might notice a deviation.

\section{Violation of FDT and effective temperature}

In order to test the violation of FDT, we use our results of Sec VIIIA scaled with Eqs. (46a) and 46b. The parameter $y(T)$ in Eqs. (46a) and 46b) measures the modification of the FDT, $2 k_{B} T \chi\left(t, t_{w}\right)=y(T) B\left(t, t_{w}\right)$ (or $\left.\tilde{\chi}=y(T) \tilde{B}\right)$, and an effective temperature ${ }^{37}$ can then defined by $T_{\text {eff }}=T / y$ (see Eq. 42].

In Fig. 26 we show a parametric plot of the scaled variables, $\tilde{\chi}(\tilde{t})$ vs. $\tilde{B}(\tilde{t})$, for a temperature $T<T_{g}$. If FDT is violated then the slope of the parametric plot should be different from unity. The parameters in Fig. 26 are $\gamma=10^{-5}$ and $T=0.02$, and different waiting times are shown. At very short rescaled times $\tilde{t}$ all curves fall onto the FDT line, while for longer times the curves asymptotically change to a different slope $y(T)$. In the inset, we show the short time regime for different $t_{w}$ values. Since the data with lower waiting time, $t_{w}=10$, is deeper in the out-of-equilibrium regime, we used the slope of $\tilde{\chi}(\tilde{B})$ with $t_{w}=10$ to compute the $y(T)$ parameter signaling the FDT violation. In Fig. 27 we show $\tilde{\chi}(\tilde{B})$ with $t_{w}=10$ at

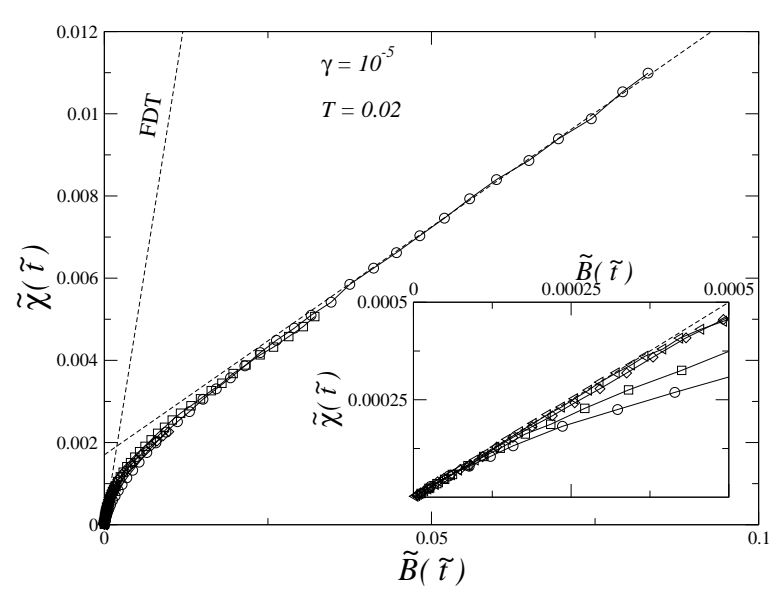

FIG. 26: Parametric plot $\tilde{\chi}(\tilde{B})$ in the VG (same data as in Fig. 24). FDT holds at short rescaled-times while a violation of FDT with $y<1$ appears at longer rescaled-times. The inset shows the short rescaled-time FDT regime for different $t_{w}$ values.

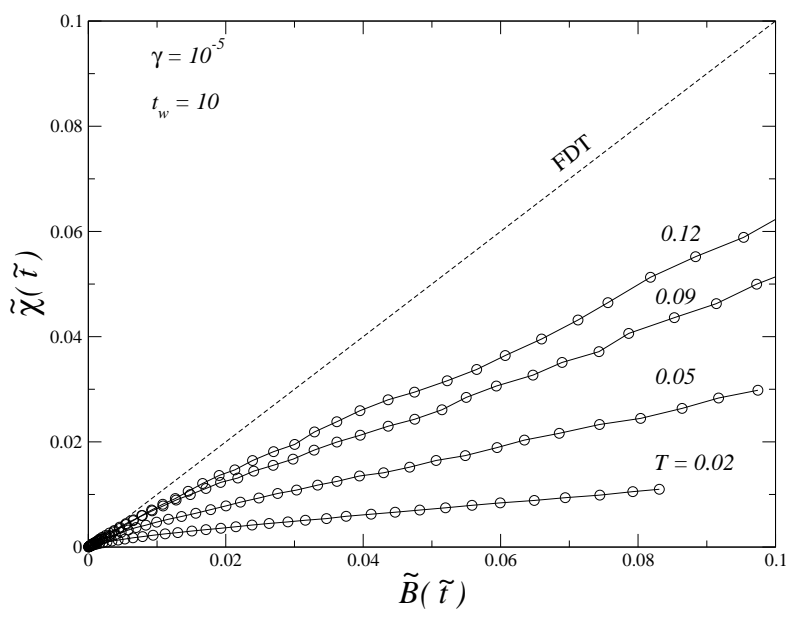

FIG. 27: Parametric plot $\tilde{\chi}(\tilde{B})$ in the VG at different temperatures given in the figure.

different temperatures; it is clear in the figure that the $y(T)$ parameter decreases with decreasing temperature. These and similar curves are used to compute the $y(T)$ values showed in Fig. 28 b). Exactly the same behavior is obtained for the single elastic line (not shown).

In order to investigate the temperature dependence of different quantities in the low temperature regime, we show the temperature variation of the scaling exponent $\alpha(T)$ and the $y(T)$ parameter in Fig. 28 We use two values of the disorder intensity, $\gamma=10^{-5}$ and $\gamma=10^{-4}$. Also shown are data for $\gamma=10^{-5}$ but for the case without in-plane interactions, quoted as single line. The values of the exponent and FDT violation factor at high temperature are $\alpha=1 / 2$, corresponding to the single elastic line without disorder limit (or VL phase), and $y=1$, corresponding to the equilibrium FDT. In 
Fig. 28 a) we see that $\alpha(T)$ depends weakly on $T$ and that it decreases with increasing disorder strength within the glassy regime. It is also observed that the in-plane interactions tend to decrease the value of $\alpha$. The parameter $y(T)$ measuring the violation of FDT rapidly increases with increasing temperature. In Fig. 28 b) we show that $y(T)=T / T_{\text {eff }}$ is well described by a linear form, implying an effective temperature $T_{\text {eff }}$ that is temperature-independent. However, a more complicated temperature-dependent effective temperature cannot be ruled out. We do not show the linear fit corresponding to $\gamma=10^{-5}$ without in-plane interactions. From the linear fit of $y(T)$ one obtains the effective temperature values quoted in the figure, $T_{\text {eff }}=0.175$ and $T_{\text {eff }}=0.203$ for $\gamma=10^{-5}$ and $\gamma=10^{-4}$, respectively.

Another feature of the scaling form (47) is the temperature dependence of the $c_{1}(T)$ and $c_{2}(T)$ coefficients. These coefficients measure the separation between the quasi-equilibrium regime and the aging regime, and hence they should converge to the same value in the equilibrium high-temperature regime. In Fig. 29 these functions are shown for the same parameters used in Fig. 28 The $T_{\text {eff }}$ values are also given in the figure. For each set of parameters $c_{1}(T)$ and $c_{2}(T)$ are closer to each other when approaching $T_{\text {eff }}$ and the high-temperature regime. This is another confirmation of the scaling form (47).

Noticeably, the obtained value of $T_{\text {eff }}=0.175$ is very close to the crossover temperature $T_{g} \approx 0.18$ below which a dynamic arrest is observed and the system can not be equilibrated, as found in Sec $\mathrm{VB}$ A similar result is observed in structural glasses: $T_{\text {eff }} \approx T_{g}$ (as in a random energy model scenario $\left.{ }^{95}\right)$.

\section{DISCUSSION}

In this paper we studied numerically the dynamics of a high-temperature superconductor model. We briefly analyzed the stationary dynamics in the VL and we focused on the outof-equilibrium dynamics of the VG. Our aim was to learn about the nature of the VG and its dynamical properties from the relaxation of different two-times correlation functions. During this study a systematic comparison with the dynamics of other glassy systems was performed.

A key feature in problems involving elastic lines, as the one we treated here, that should be highlighted is the relevance of finite size effects. By comparing the evolution of the roughness and dynamic wandering in the vortex system to the ones in the EW equation for growing interfaces ${ }^{89}$ we identified three dynamic regimes present at all interesting temperatures: $(i)$ a very short time-difference normal diffusion regime without effective elastic interactions; (ii) a sub-diffusion intermediate time-difference regime characterized by a growing longitudinal correlation length; (iii) a long time-difference regime where the correlation length has reached the system size and normal diffusion simply reflects the center of mass diffusion. In order to separate aging effects from finite size effects we constrained the remaining study to regimes $(i)$ and (ii), i.e. before roughness saturation.

Strikingly, the behavior of the density-density correlation

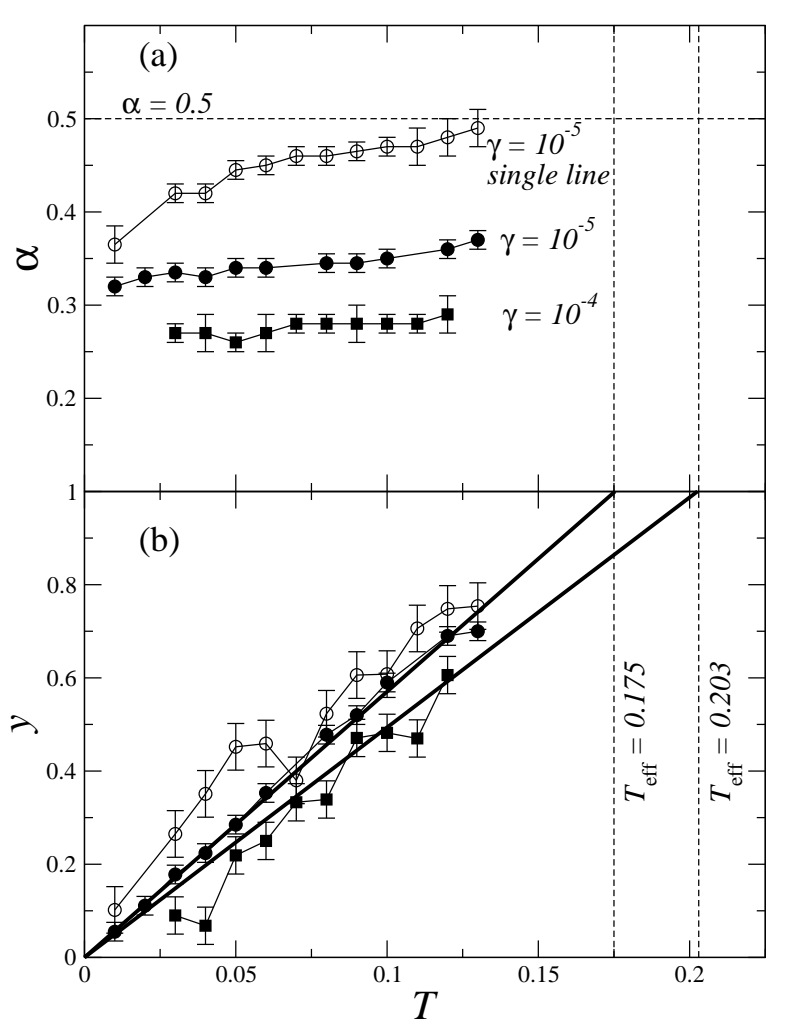

FIG. 28: (a) The scaling exponent $\alpha(T)$ and (b) the $y(T)$ parameter measuring the FDT violation in the VG with two disorder intensities, $\gamma=10^{-5}$ and $\gamma=10^{-4}$. Data for a single elastic line in a random environment with $\gamma=10^{-5}$ are also shown. The effective temperature $T_{\text {eff }}$ obtained from linear fits $y(T)=T / T_{\text {eff }}$ is given for the cases $\gamma=10^{-5}$ and $\gamma=10^{-4}$.

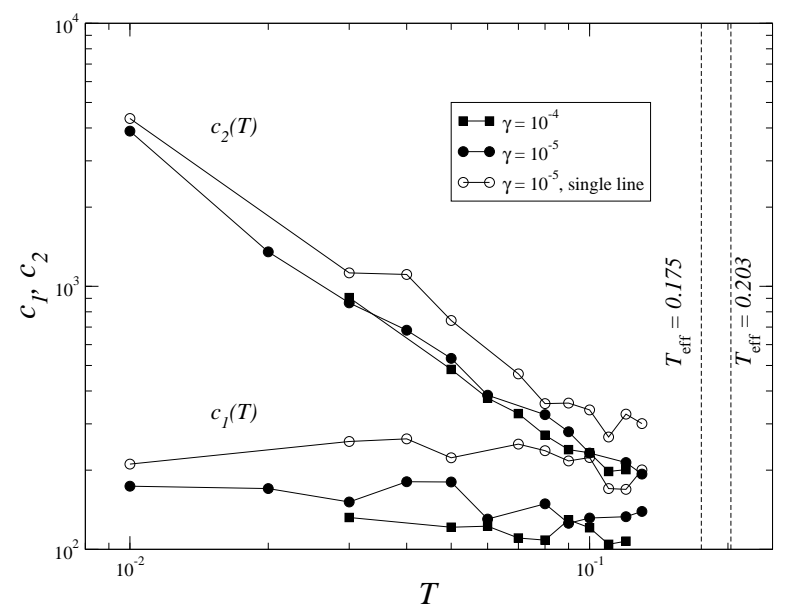

FIG. 29: Evolution of the coefficients $c_{1}(T)$ and $c_{2}(T)$ with temperature for the same parameters as in Fig. 28] They tend to the same value with increasing temperature, as suggested by the scaling form 47. 
function $C_{k}\left(t, t_{w}\right)$ resembles in many aspects the one observed in simulations of Lennard-Jones glass formers ${ }^{62}$ In particular, the wave-vector dependence of the correlation follows the same general trend. The main difference is that the densitydensity correlation does not develop a well defined plateau for long waiting times in the VG, while it seems to do in glassforming liquids. This is not due to the use of extremely short waiting times as could be expected in an additive aging scaling, but it is in the core of the multiplicative aging scaling we found in the present simulations.

We showed that the two-times evolution of the pancake mean-squared-displacement $B\left(t, t_{w}\right)$ is very well described by the multiplicative scaling diffusive-aging scenario earlier proposed in the numerical study of the out-of-equilibrium dynamics of the directed polymer in random media model, ${ }^{71}$ found analytically in the massless scalar field in $D=1$ (related to the Edwards-Wilkinson surface), and obtained numerically and analytically in Sinai diffusion ${ }^{75,76,77}$ It is interesting to stress that the model of a $D$-dimensional manifold embedded in an $N$-dimensional space under the effect of a combined disordered and harmonically confining potential - a toy model that is usually used to model vortex systems - fails to capture the multiplicative scaling in the large $N$ limit ${ }^{73.74}$ This model has, instead, a well defined transition at a finite temperature below which correlations and responses scale in an additive way with a stable plateau.

We studied the violations of the FDT out of equilibrium. We constructed the parametric plot of linear integrated response against displacement after having eliminated the power of the waiting-time, $t_{w}^{\alpha}$, that appears multiplying these quantities. The resulting plot is linear, at least within the accuracy of out data, and it allowed us to identify an effective temperature as the slope of the linear plot. The same behavior was found in the lattice model of the directed polymer in a random environment ${ }^{69.70}$ The relation between integrated response and displacement, once the factors $t_{w}^{\alpha}$ have been taken into account, might involve though a non-trivial function of the displacement itself, not visible within the accuracy of our data, as has been found, for instance, in the 2D scalar field and XY model ${ }^{64.65}$ The FDT violation in the 'mean-field' model of a relaxing $D$-dimensional manifold in an infinitedimensional embedding space ${ }^{73,74}$ with a short-ranged disorder potential has this type of violation of FDT though it is not necessary in this case to eliminate additional $t_{w}^{ \pm \alpha}$ factors.

We performed the analysis of displacement, correlations and responses at several low temperatures and we analysed the temperature dependence of the exponent $\alpha(T)$ as well as the coefficients $c_{1}(T)$ and $c_{2}(T)$ that characterize the scaling function. We also followed the evolution with temperature of the parameter signaling the FDT violation, $y(T)$. We considered two intensities of the pinning disorder and we compared the data to the a priori simpler case of a single line without in-plane interactions. All the parameters, $y(T), \alpha(T), c_{1}(T)$ and $c_{2}(T)$ show a clear trend to reach the high-temperature behavior: $y(T)=1, \alpha(T)=1 / 2$, and $c_{1}(T)=c_{2}(T)$ when approaching the VL from the glassy regime. From these outof-equilibrium measurements the crossover temperature was found to be very similar to the value of the effective tempera- ture, $T_{\text {eff }}$, obtained from the fit of the FDT violation at very long time-differences to a linear form with $y(T)=T / T_{\text {eff }}$. This result is similar to what happens in the random-energy model in which the effective temperature takes the transition value in the full low-temperature phase.

To further test the meaning of the crossover temperature and the effective temperature $T_{\text {eff }}$, we studied the dynamic arrest approaching the VG from the VL. By fitting a mean-squareddisplacement correlation, taking into account sub-linear diffusion, we determined a relaxation time $t_{r}$. The so-obtained relaxation time increases with temperature as $t_{r} \sim T^{-2}$, at rather high temperatures, as expected in disordered free dynamics. However, below a characteristic temperature a sudden further increase of the relaxation time $t_{r}$ was found. We identified the crossover temperature between normal relaxation and rapidly growing relaxation with a glass temperature $T_{g}$. When comparing with the effective temperature we found that both temperatures are of the same order, a fact already observed in other glassy systems. This suggest a freezing of the slow degrees of freedom of the system around the crossover temperature.

One outstanding result of our simulations is that all the outof-equilibrium properties are mainly dictated by the elastic line energy, i.e. they are all observable in the single line without in-plane interactions. This means that for the parameters used in our simulations the two relevant competing energies are the elastic line and pinning energies. This fact points out that the relevant dynamic properties are also observed in simplified models of elastic lines in disordered media as directed polymers ${ }^{71,72.90}$ or continuous models similar to that studied here ${ }^{49.101}$ It is worth mentioning that this cannot be the case at very high magnetic fields, i.e. very high vortex density, since at some point the in-plane interaction should become the relevant energy scale. In this condition strong excluded volume effects, such as the "cage effect" in supercooled liquids, should be appreciable. A simplified model in this direction was considered by Petäjä et al., who considered elastic lines with hard-core interactions in random environments. ${ }^{97}$

From our findings several extensions could be envisaged. Concerning the possible experimental observation of the outof-equilibrium dynamics of the VG phase, new experiments monitoring magnetic relaxation 44.45 would be most welcome. To measure aging, one possibility is to perform transport experiments following a protocol similar to the one used by Ovadyahu and coworkers to study the electron glass regime in Anderson insulators 102.103 Tests of FDT would require simultaneous measurements of noise (magnetic and/or voltage noise) and time dependent response (relaxation of magnetization and/or resistivity); similar tests have been performed in other systems $104,105,106$ Another possibility would be to test the aging dynamics and the FDT violation in vortex shear experiments ${ }^{107.108}$ It is well known that in shear-thinning systems the time scale introduced by the shear rate basically replaces the waiting time; at lower shear rates the relaxation is longer, i.e. the system "ages" with decreasing shear rate 109.110 .111 .112 Still another possibility is to measure effective temperatures using a tracer embedded in the vortex systems. Finally, the out-of-equilibrium dynamics of the center 
of mass diffusion could be experimentally tested.

It would also be interesting to study the out-of-equilibrium relaxation of the $\mathrm{BG}$ and compare the outcome to the results obtained in this work for the VG.

\section{Acknowledgments}

We thank C. Chamon, A. B. Kolton, T. Giamarchi, J. L. Iguain and $\mathrm{H}$. Yoshino for very useful discussions and $\mathrm{H}$. Yoshino for access to his unpublished results on the dynamics of a directed polymer in a random environment. We acknowledge financial support from SECYT-ECOS P. A01E01 (SB, LFC DD); Conicet PIP2005-5596, CNEA P5-PID-93-7, ANPCYT PICT99-03-06343, and ICTP grant NET-61 (SB and DD); Fundación Antorchas (SB) and PICS 3172 (LFC). SB thanks the LPTHE Jussieu (France), SB and LFC the Universidad Nacional de Mar del Plata (Argentina), and LFC the Newton Institute for Mathematical Sciences (UK) for hospitality during the preparation of this work. LFC is a member of Institut Universitaire de France.
1 G. Blatter, M. V. Feigel'man, V. B. Geshkenbein, A. I. Larkin, and V. M. Vinokur, Rev. Mod. Phys. 66, 1973 (1994).

2 H. Brandt, Rep. Prog. Phys. 58, 1465 (1995).

3 L. F. Cohen and H. J. Jensen, Rep. Prog. Phys. 60, 1581 (1997).

4 T. Nattermann and S. Scheidl, Adv. in Phys. 49, 607 (2000).

5 A. I. Larkin and Y. N. Ovchinnikov, Zh. Eksp. Teor. Fiz. 65, 1704 (1973 [Sov. Phys. JETP 38, 854 (1974)]).

6 D. G. Grier, C. A. Murray, C. A. Bolle, P. L. Gammel, D. J. Bishop, D. B. Mitzi, and A. Kapitulnik, Phys. Rev. Lett. 66, 2270 (1991).

7 R. Cubitt, E. M. Forgan, G. Yang, S. L. Lee, D. M. Paul, H. A. Mook, M. Yethiraj, P. H. Kes, T. W. Li, A. A. Menovsky, et al., Nature (London) 365, 407 (1993).

8 T. Giamarchi and P. Le Doussal, Phys. Rev. Lett. 72, 1530 (1994).

9 T. Giamarchi and P. Le Doussal, Phys. Rev. B 52, 1242 (1995).

10 M. P. A. Fisher, Phys. Rev. Lett. 62, 1415 (1989).

11 D. S. Fisher, M. P. A. Fisher, and D. A. Huse, Phys. Rev. B 43, 130 (1991).

12 H. Safar, P. L. Gammel, D. A. Huse, D. J. Bishop, W. C. Lee, J. Giapintzakis, and D. M. Ginsberg, Phys. Rev. Lett. 70, 3800 (1993).

13 W. K. Kwok, J. A. Fendrich, C. J. van der Beek, and G. W. Crabtree, Phys. Rev. Lett. 73, 2614 (1994).

14 J. D. Reger, T. A. Tokuyasu, A. P. Young, and M. P. A. Fisher, Phys. Rev. B 44, 7147 (1991).

15 H. S. Bokil and A. P. Young, Phys. Rev. Lett. 74, 3021 (1995).

16 C. Wengel and A. P. Young, Phys. Rev. B 54, R6869 (1996).

17 C. Wengel and A. P. Young, Phys. Rev. B 56, 5918 (1997).

18 J. Kisker and H. Rieger, Phys. Rev. B 58, 8873 (1998).

19 A. van Otterlo, R. T. Scalettar, and G. T. Zimányi, Phys. Rev. Lett. 81, 1497 (1998).

20 T. Olson and A. P. Young, Phys. Rev. B 61, 12467 (2000).

21 C. Reichhardt, A. van Otterlo, and G. T. Zimányi, Phys. Rev. Lett. 84, 1994 (2000).

22 J. Kierfeld and V. M. Vinokur, Phys. Rev. B 61, R14928 (2000).

23 D. Li and B. Rosenstein, Phys. Rev. Lett. 90, 167004 (2003).

24 J. Lidmar, Phys. Rev. Lett. 91, 097001 (2003).

25 K. A. Müller, M. Takashige, and J. G. Bednorz, Phys. Rev. Lett. 58, 1143 (1987).

26 Y. Yeshurum and A. P. Malozemoff, Phys. Rev. Lett. 60, 2202 (1988)

27 J. Deak, M. McElfresh, J. R. Clem, Z. Hao, M. Konczykowski, R. Muenchausen, S. Foltyn, and R. Dye, Phys. Rev. B 49, 6270 (1994).

28 R. H. Koch, V. Foglietti, W. J. Gallagher, G. Koren, A. Gupta, and M. P. A. Fisher, Phys. Rev. Lett. 63, 1511 (1989).
29 A. M. Petrean, L. M. Paulius, W.-K. Kwok, J. A. Fendrich, and G. W. Crabtree, Phys. Rev. Lett. 84, 5852 (2000).

30 P. Olsson, Phys. Rev. Lett. 91, 077002 (2003).

31 W. Jiang, N.-C. Yeh, T. A. Tombrello, A. P. Rice, and F. Holtzberg, J. Phys.: Condens. Matter 9, 8085 (1997).

32 D. R. Strachan, M. C. Sullivan, P. Fournier, S. P. Pai, T. Venkatesan, and C. J. Lobb, Phys. Rev. Lett. 87, 067007 (2001).

33 M. D. Ediger, C. A. Angell, and S. R. Nagel, J. Phys. Chem. 100, 13200 (1996).

34 E. Vincent, J. Hammann, M. Ocio, J.-P. Bouchaud, and L. F. Cugliandolo, in Complex Behaviour of Glassy Systems, edited by E. Rubi (Springer-Verlag, Berlin, 1996).

35 L. F. Cugliandolo, in Slow Relaxations and Nonequilibrium Dynamics in Condensed Matter, edited by J. L. Barrat, J. Dalibard, M. Feigel'man, and J. Kurchan (Springer, Berlin, 2002).

36 A. Crisanti and F. Ritort, J. Phys. A 36, 181 (2003).

37 L. F. Cugliandolo, J. Kurchan, and L. Peliti, Phys. Rev. E 55, 3898 (1997).

38 A. B. Kolton, R. Exartier, L. F. Cugliandolo, D. Domínguez, and N. Grønbech-Jensen, Phys. Rev. Lett. 89, 227001 (2002).

39 B. Sas, F. Portier, K. Vad, B. Keszei, L. F. Kiss, N. Hegman, S. M. I. Puha, and F. I. B. Williams, Phys. Rev. B 61, 9118 (2000).

40 F. Portier, G. Kriza, B. Sas, L. F. Kiss, I. Pethes, K. Vad, B. Keszei, and F. I. B. Williams, Phys. Rev. B 66, 140511 (2002).

41 R. Exartier and L. F. Cugliandolo, Phys. Rev. B 66, 012517 (2002).

42 C. J. Olson, C. Reichhardt, R. T. Scalettar, G. T. Zimányi, and N. Grønbech-Jensen, Phys. Rev. B 67, 184523 (2000).

43 S. O. Valenzuela and V. Bekeris, Phys. Rev. Lett. 84, 4200 (2000).

44 E. L. Papadopoulou, P. Nordblad, P. Svendlindh, R. Schöneberger, and R. Gross, Phys. Rev. Lett. 82, 173 (1999).

45 E. L. Papadopoulou, P. Svendlindh, and P. Nordblad, Phys. Rev. B 65, 144524 (2002).

46 M. S. Li, P. Nordblad, and H. Kawamura, Phys. Rev. Lett. 86, 1339 (2001).

47 M. Nicodemi and H. J. Jensen, Phys. Rev. B 65, 144517 (2002).

48 M. Nicodemi and H. J. Jensen, Phys. Rev. Lett. 86, 4378 (2001).

49 A. B. Kolton, A. Rosso, and T. Giamarchi, Phys. Rev. Lett. 94, 047002 (2005).

50 S. Bustingorry, L. F. Cugliandolo, and D. Domínguez, Phys. Rev. Lett. 96, 027001 (2006).

51 A. A. Abrikosov, Sov. Phys. JETP. 5, 1147 (1957).

52 P. G. De Gennes, Superconductivity of Metals and Alloys (W. A. Benjamin, 1966, New York). 
53 M. Tinkham, Introduction to Superconductivity (Mc Graw Hill, 1975, New York).

54 T. Giamarchi and P. Le Doussal, Phys. Rev. B 55, 6577 (1997).

55 K. Deligiannis, P. A. J. de Groot, M. Oussena, S. Pinfold, R. Langan, R. Gagnon, and L. Taillefer, Phys. Rev. Lett. 79, 2121 (1997).

56 E. Zeldov, D. Majer, M. Konczykowski, V. B. Geshkenbein, and V. M. V. H. Shtrikman, Nature (London) 375, 373 (1994).

57 T. Klein, I. Joumard, S. Blanchard, J. Marcus, S. Cubitt, T. Giamarchi, and P. Le Doussal, Nature (London) 413, 404 (2001).

58 Y. Nonomura and X. Hu, Phys. Rev. Lett. 86, 5140 (2001).

59 P. Olsson and S. Teitel, Phys. Rev. Lett. 87, 137001 (2001).

60 M. V. Feigel'man, V. B. Geshkenbein, A. I. Larkin, and V. M. Vinokur, Phys. Rev. Lett. 63, 2303 (1989).

61 T. Nattermann, Phys. Rev. Lett. 64, 2454 (1990).

62 W. Kob and J.-L. Barrat, Eur. Phys. J. B 13, 319 (2000).

63 V. Viasnoff and F. Lequeux, Phys. Rev. Lett. 89, 065701 (2002).

64 L. F. Cugliandolo, J. Kurchan, and G. Parisi, J. Phys. I (France) 4, 1641 (1994).

65 L. Berthier, P. C. W. Holdsworth, and M. Sellitto, J. Phys. A 34, 1805 (2001).

66 A. Gambassi and P. Calabrese, J. Phys. A 38, 133 (2005).

67 M. Henkel and M. Pleimling, Europhys. Lett. 69, 524 (2005).

68 C. Godrèche and J.-M. Luck, J. Phys. C (Solid St. Phys.) 14, 1589 (2002).

69 A. Barrat, Phys. Rev. E 55, 5651 (1997).

70 H. Yoshino, J. Phys. A 29, 1421 (1996).

71 H. Yoshino, Phys. Rev. Lett. 81, 1493 (1998).

72 S. Bustingorry, J. L. Iguain, C. Chamon, L. F. Cugliandolo, D. Domínguez (unpublished).

73 L. F. Cugliandolo and P. Le Doussal, Phys. Rev. E 53, 1525 (1996).

74 L. F. Cugliandolo, J. Kurchan, and P. Le Doussal, Phys. Rev. Lett. 76, 2390 (1996).

75 L. Laloux and P. Le Doussal, Phys. Rev. E 57, 6296 (1998).

76 D. S. Fisher, P. Le Doussal, and C. Monthus, Phys. Rev. Lett. 80, 3539 (1998).

77 P. Le Doussal, C. Monthus, and D. S. Fisher, Phys. Rev. E 59, 4795 (1999).

78 D. Nelson, Phys. Rev. Lett. 60, 1973 (1988).

79 D. Nelson and H. S. Seung, Phys. Rev. B 39, 9153 (1989).

80 D. Nelson and V. M. Vinokur, Phys. Rev. Lett. 68, 2398 (1992).

81 S. Ryu and D. Stroud, Phys. Rev. B 54, 1320 (96).

82 H. Nordborg and G. Blatter, Phys. Rev. B 58, 14556 (1998).

83 A. V. Lopatin and V. M. Vinokur, Phys. Rev. Lett. 92, 067008 (2004).

84 J. Kierfeld and V. M. Vinokur, Phys. Rev. Lett. 94, 077005
(2005)

85 A. van Otterlo, R. T. Scalettar, G. T. Zimányi, R. Olsson, A. Petrean, W. Kwok, and V. Vinokur, Phys. Rev. Lett. 84, 2493 (2000).

86 See http://fftw.org/.

87 J. R. Clem, Phys. Rev. B 43, 7837 (1991).

88 C. J. Olson, G. T. Zimányi, A. B. Kolton, and N. GrønbechJensen, Phys. Rev. Lett. 85, 5416 (2000).

89 A.-L. Barabási and H. E. Stanley, Fractal Concepts in Surface Growth (Cambridge University Press, Cambridge, 1995).

$90 \mathrm{H}$. Yoshino (unpublished).

91 S. F. Edwards and D. R. Wilkinson, Proc. R. Soc. London, Ser. A 381, 17 (1982).

92 H.-N. Yang, T.-M. Lu, and G.-C. Wang, Phys. Rev. Lett. 68, 2612 (1992).

93 Y.-L. He, H.-N. Yang, T.-M. Lu, and G.-C. Wang, Phys. Rev. Lett. 69, 3770 (1992).

94 H.-N. Yang, T.-M. Lu, and G.-C. Wang, Phys. Rev. B 47, 3911 (1993).

95 G. Parisi, Phys. Rev. Lett. 79, 3660 (1997).

96 F. Family and T. Vicsek, J. Phys. A 18, L75 (1985).

97 V. Petäjä, D.-S. Lee, M. Alava, and H. Rieger, J. Stat. Mech. p. P10010 (2004).

98 T. Halpin-Healy and Y.-C. Zhang, Phys. Rep. 254, 215 (1995).

99 A. J. Bray, Adv. in Phys. 43, 357 (1994).

100 F. Krzakala, Phys. Rev. Lett. 94, 077204 (2005).

101 A. B. Kolton, A. Rosso, and T. Giamarchi, Phys. Rev. Lett. 95, 180604 (2005).

102 A. Vaknin, Z. Ovadyahu, and M.Pollak, Phys. Rev. Lett. 84, 3402 (2000).

103 A. Vaknin, Z. Ovadyahu, and M.Pollak, Phys. Rev. B 65, 134208 (2002)

104 S. Grigera and N. Israeloff, Phys. Rev. Lett. 83, 5038 (1999).

105 L. Bellon, S. Ciliberto, and C. Laroche, Europhys. Lett. 53, 511 (2000).

106 D. Hérisson and M. Ocio, Phys. Rev. Lett. 88, 257202 (2002).

107 D. López, W. K. Kwok, H. Safar, R. J. Olsson, A. M. Petrean, L. Paulius, and G. W. Crabtree, Phys. Rev. Lett. 82, 1277 (1999).

108 A. A. B. Brojeny and J. R. Clem, Phys. Rev. B 64, 184507 (2001).

109 L. Berthier, J.-L. Barrat, and J. Kurchan, Phys. Rev. E 61, 5464 (2000).

110 J.-L. Barrat and L. Berthier, Phys. Rev. E 63, 012503 (2000).

111 L. Berthier and J.-L. Barrat, Phys. Rev. Lett. 89, 095702 (2002).

112 L. Berthier and J.-L. Barrat, J. Chem. Phys. 116, 6228 (2002). 